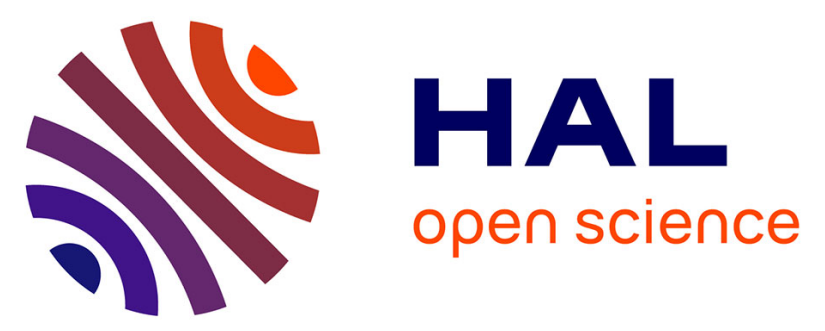

\title{
Chemical ionization of carboxylic acids and esters in negative mode selected ion flow tube - Mass spectrometry (SIFT-MS)
}

Mylène Ghislain, Marine Reyrolle, Jean-Marc Sotiropoulos, Thierry Pigot, Mickael Le Béchec

\section{To cite this version:}

Mylène Ghislain, Marine Reyrolle, Jean-Marc Sotiropoulos, Thierry Pigot, Mickael Le Béchec. Chemical ionization of carboxylic acids and esters in negative mode selected ion flow tube - Mass spectrometry (SIFT-MS). Microchemical Journal, 2021, 169, pp.106609. 10.1016/j.microc.2021.106609 . hal-03301604

\section{HAL Id: hal-03301604 https://hal.mines-ales.fr/hal-03301604}

Submitted on 30 Aug 2021

HAL is a multi-disciplinary open access archive for the deposit and dissemination of scientific research documents, whether they are published or not. The documents may come from teaching and research institutions in France or abroad, or from public or private research centers.
L'archive ouverte pluridisciplinaire HAL, est destinée au dépôt et à la diffusion de documents scientifiques de niveau recherche, publiés ou non, émanant des établissements d'enseignement et de recherche français ou étrangers, des laboratoires publics ou privés. 


\title{
Chemical ionization of carboxylic acids and esters in negative mode selected ion flow tube - mass spectrometry (SIFT-MS)
}

\author{
Mylène Ghislain, ${ }^{\text {a }}$ Marine Reyrolle, ${ }^{\mathrm{a}}$ Jean-Marc Sotiropoulos, ${ }^{\text {a }}$ Thierry Pigot, ${ }^{\mathrm{a}}$ Mickael \\ Le Bechec ${ }^{\text {a* }}$ \\ ${ }^{\text {a }}$ Universite de Pau et des Pays de 1'Adour, E2S UPPA, CNRS, IMT Mines Ales, IPREM, Pau, \\ France \\ * Correspondence to : Mickael Le Bechec, Universite de Pau et des Pays de l'Adour, E2S \\ UPPA, CNRS, IPREM, Institut des sciences analytiques et de Physicochimie pour \\ l'environnement et les Matériaux, UMR5254, Hélioparc, 2 avenue Président Angot, 64053, \\ PAU cedex 9, France \\ e-mail : mickael.lebechec@univ-pau.fr
}

\begin{abstract}
Selected ion flow tube - mass spectrometry (SIFT-MS) is based on chemical reactivity of analytes with reactant ions. The complete understanding of all the ion-molecule reactions is the key to the deployment of this technology for the direct and rapid analysis of VOCs in the gas phase. In the present work, we focused on direct analysis of carboxylic acids and esters, compounds involved in many biochemical processes.

The ion-molecule reactions of negative precursor ions $\left(\mathrm{OH}^{-}, \mathrm{O}^{\bullet-}, \mathrm{O}_{2}{ }^{\bullet-}, \mathrm{NO}_{2}{ }^{-}\right.$and $\left.\mathrm{NO}_{3}{ }^{-}\right)$with 9 carboxylic acids and 21 esters were investigated in order to provide product ions and rate constants for these compounds analysis by SIFT-MS. The modelling of ion-molecule reaction paths by $a b$-initio calculations supported the experimental results and provided a better understanding of these gas phase reactions.

The most common reaction mechanism with all the tested molecules is deprotonation. However, other specific reactivities, leading to the formation of anion-molecule complexes with $\mathrm{NO}_{2}{ }^{-}$and $\mathrm{O}_{2}{ }^{-}$in particular, are observed. Furthermore, specific fragmentations of chemical families are also noticed and confirmed with $\mathrm{O}_{2}{ }^{-}$and $\mathrm{O}^{-}$. Finally, we demonstrated that the composition of the matrix $\left(\mathrm{H}_{2} \mathrm{O}, \mathrm{CO}_{2}\right)$ participates in the formation of secondary ions.

In this work, we determined the kinetic rate constants in the nitrogen carrier gas and the detection limits of all the compounds with the eight precursor ions used by SIFT-MS, allowing accurate quantification in the gas phase at the ppb level. These results contribute to enlarge the negative ionization SIFT-MS measurements to applications where quantification of organic acids and esters are required.
\end{abstract}

\section{Keywords}

Selected Ion Flow Tube - Mass Spectrometry; SIFT-MS; negative ionization; volatile fatty acids; esters; modeling 


\section{Introduction}

The analysis of volatile organic compounds (VOCs) in the gas phase is a crucial point in many application fields. In environmental issues or indoor air quality, VOCs are considered as pollutants to be monitored or indicators of pollution events. Due to their health impact, regulations require their monitoring to assess their concentrations and emission rates, sometimes at trace levels [1,2]. For medical or biological application fields, VOCs are no longer studied as pollutants but as markers of interest $[3,4]$. The emerging discipline volatolomics consists in the analysis of volatile compounds emitted by a biological system [5]. Thus, the specific monitoring of these biomarkers can provide essential information on the studied biological process. Volatolomics can be applied to health issues as well as in the food industry, or to more fundamental research fields [6]. VOCs monitoring is currently used as non-invasive approach to disease diagnosis such as cancers, asthma, diabetes or epilepsy and many studies show specific volatile markers of these diseases in the exhaled air, blood or urine of patients [5,7-14]. Very recently, a study revealed a characteristic VOCs profile in exhaled air of COVID-19 patients [15]. In the food industry, VOCs constitute an actual chemical fingerprint of products reflecting their organoleptic qualities, their evolution and conservation [16,17]. Specific VOCs can also be tracked to monitor processes occurring in, for example, butyric and acetic acids in cheeses fermentation drift [18]. VOCs gas phase profiles are also key parameters in microbiology field and provide a lot of information about microbiological imbalances or contaminations, synthesis of metabolites or microbial identifications [19-22].

Thus, the need for VOCs measurement is increasing with the number of application fields with ever more requirements and challenges in terms of sensitivity, range of compounds in often complex mixtures and sampling constraints, as well as speed rates of results. Direct-injection mass spectrometric (DIMS) technologies such as atmospheric-pressure chemical ionization (APCI), proton-transfer reaction mass spectrometry (PTR-MS) or selected ion flow tube mass spectrometry (SIFT-MS), offer relevant solutions to rapid monitoring and quantification of VOCs for all these applications. These technologies combining sensitivity, robustness and speed with time resolution meet these new constraints. In addition, these non-specific techniques allow the analysis of a wide range of volatile compounds, not restricted to organic ones.

Direct-injection mass spectrometry is based on chemical reactions between the analytes and precursor ions [23]. Understanding ion-molecule reactions is nowadays a key point for DIMS analysis. Knowledge of product ions formed, together with the kinetic data, is essential for the quantification of target compounds as well as for the elucidation of complex spectra. While reactivity in positive ionization is well documented, in particular with the $\mathrm{H}_{3} \mathrm{O}^{+}$precursor ion, data on negative ionization remain sparse. However, negative ionization in direct injection techniques is developing more and more and further multiplies the possibilities of analysis. The recent development of commercial instruments using a source of negative ions leads to a growing interest in anion-molecule reactivity [24].

Anion reactivity is generally well known but specific data focused on target compounds families are still lacking [24]. Data sparsely appear in the literature to gradually build a database on negative ionization gas phase chemistry. Data on aldehydes are now available [25-27], as well as on alcohols [6,7], ketones [26,28], fumigants, acid gases [29], ozone [30,31] and carbon dioxide $[29,32]$.

In order to contribute to the enhancement of this database, the present work focus on the study of the SIFT-MS reactivity of aliphatic carboxylic acids and esters with negative reagent ions $\left(\mathrm{OH}^{-}, \mathrm{O}^{\bullet-}, \mathrm{O}_{2}{ }^{\bullet-}, \mathrm{NO}_{2}^{-}\right.$and $\left.\mathrm{NO}_{3}^{-}\right)$. Both chemical families are widely found as pollutants and 
biological samples, most of the time in a complex mixture of volatiles. Accurate analyses were carried out with SIFT-MS Voice 200 ultra for each individual pure compound in controlled matrices. These experimental measurements were combined with a theoretical approach: while the experimental work allows the identification of product ions from reaction of carboxylic acids and esters with the various negative precursor ions, the calculation of reactions paths and energies allows a consideration of the most likely reactions leading to the formation of the experimentally detected product ions. Experimental kinetic data are complemented by calculation of theoretical collision rate constants. To the best of our knowledge, this study is the first systematic study of these families by negative ionization with a SIFT-MS.

\section{Experimental section}

1 - Selected ion flow tube - Mass spectrometry (SIFT-MS)

A commercial SIFT-MS apparatus (Voice 200 Ultra, SYFT Technologies, Christchurch, New Zealand) with heated High Performance Inlet (HPI) was used to study the gas phase chemical reactivity of carboxylic acids and esters with negative reagent ions $\mathrm{OH}^{-}, \mathrm{O}^{\bullet-}, \mathrm{O}_{2}{ }^{\bullet-}$, $\mathrm{NO}_{2}{ }^{-}$and $\mathrm{NO}_{3}{ }^{-}$[29]. Each reagent was studied individually since the upstream quadrupole mass filter selects the precursor ions entering the flow tube. The device is equipped with a dual source so that a positive ionization mode using $\mathrm{H}_{3} \mathrm{O}^{+}, \mathrm{NO}^{+}$and $\mathrm{O}_{2}{ }^{+}$precursor ions is also available. The LabSyft 1.6.2 software was also supplied by SYFT Technologies.

Chemicals were purchased from Sigma-Aldrich Inc. (USA), with $>97 \%$ of purity (except Formic acid $95 \%$ of purity). Carboxylic acids and esters pure gaseous atmospheres were introduced in the flow tube through an inlet line heated at $373 \mathrm{~K}$ at a flow rate of $20 \mathrm{~mL} \mathrm{~min}^{-1}$. The reactivity was studied at a temperature of $393 \mathrm{~K}$ and a pressure of 0.48 torr in pure $\mathrm{N}_{2}$ (from Air liquid, Alphagaz 2, $\geq 99.9999 \%$ ) (flow tube conditions).

All the product ions are monitored and counted by a second quadrupole mass filter mounted downstream of the flow tube.

For a reaction between a precursor ion $\mathrm{R}$ and an analyte $\mathrm{A}$ leading to the formation of a product ion $\mathrm{P}$ with a reaction rate constant $k$, the quantification by SIFT-MS only requires the measurement of the precursor ion $[R]$ and the product ion $[P]$ count rates in the flow tube $\left(\right.$ count $\left.\mathrm{s}^{-1}\right)$, and external calibration is not needed. In large excess of precursor ions $([R]>>[P])$, the analyte number density $[A]$ into the flow tube can be expressed:

$$
[A]=\frac{I C F_{P} \times[P]}{I C F_{R} \times[R] k t_{r}}
$$

with $t_{r}$ the reaction time in the flow tube (s), ICF $F_{P}$ the instrument correction factor of product ion and $I C F_{R}$ the instrument correction factor of precursor ion. These last two factors are used to consider the influence of ionic diffusion and mass discrimination [33].

\section{2 - Generation of carboxylic acids and esters atmospheres}

Gaseous atmospheres of the analytes are generated by spiking aqueous solutions prepared from pure liquid individual compound $(2 \mu \mathrm{L}$ ), into $1 \mathrm{~L}$ glass bottles (Duran, Mainz, Germany) filled with dry nitrogen via a septum. The bottles were closed with screw caps with three GL14 ports (Duran) equipped with one septum and two plugged 1/4-inch PFA tubes 
(Swagelock Company, Ohio USA). The bottles were heated to $60^{\circ} \mathrm{C}$; after 2 hours of incubation, the injected amounts are fully vaporized. These heating conditions were previously validated with GC-FID measurements.

The SIFT-MS sampling was carried out at the outlet of the bottles at a flow rate of $20 \mathrm{~mL} \mathrm{~min}^{-}$ 1. A Tedlar bag (Zefon International Inc., Ocala, FL, USA) filled with dry nitrogen was connected at the inlet to compensate the depression into the bottle during sampling. The bottles were maintained at $60^{\circ} \mathrm{C}$ during the sampling.

Aqueous solutions were prepared so that final concentrations in the $1 \mathrm{~L}$ bottles were between several hundred ppbV and a few ppmV in order to be under the optimum conditions for SIFTMS detection and quantification (i.e. above the limit of quantification and without overconsumption of precursor ions). SIFT-MS quantification in positive mode (with $\mathrm{H}_{3} \mathrm{O}^{+}, \mathrm{NO}^{+}$ and $\mathrm{O}_{2}{ }^{+}$precursor ions) was used to monitor the generated concentration.

Previous studies showed that the air matrix can affect the reactions in the flow tube due to the introduction of oxygen, carbon dioxide and water along with the compound [28,32,34]. In order to study the influence of the matrix on ion-molecule reactions, gaseous atmospheres of carboxylic acids and esters were also generated in different synthetic air matrices with or without dioxygen, carbon dioxide and water, using a gas dilution setup (Serv'Instrumentation) and gas tank (Air liquid)[32].

3 - Experimental study of ion-molecule reactions

Full scan mass spectra were recorded sequentially for each negative precursor ions $\left(\mathrm{OH}^{-}\right.$ , $\mathrm{O}^{\bullet-}, \mathrm{O}_{2}{ }^{\bullet-}, \mathrm{NO}_{2}{ }^{-}$and $\mathrm{NO}_{3}{ }^{-}$) in a $\mathrm{m} / \mathrm{z}$ range of 15 to 250 with an integration time of $120 \mathrm{~s}$. First, data acquisition on blank matrices were carried out to measure the background signal. The contribution of water, dioxygen and carbon dioxide on the mass spectrum is known and documented for each negative precursor ions [28,32]. Then each compound was scanned individually in different matrices and at different concentration levels. Separate samples were prepared for each precursor ions.

In nitrogen matrix, the signals depending on the analyte concentration provide information on the product ions resulting from the reaction between the analyte and the precursor ion. Moreover, the signals depending on the presence of water, dioxygen or $\mathrm{CO}_{2}$ also inform on the reactivity of the analyte with the latter or on secondary reactions between product ions and water, dioxygen or carbon dioxide molecules.

\section{4 - Experimental rate constants}

Experimental rate constants $(k)$ are determined from fixed concentrations of carboxylic acids and esters in dry nitrogen matrix (the amount of water in the aqueous solutions injected into the bottle is considered negligible). As $[P],[R]$ and $t_{r}$ are measured parameters, at known concentration, experimental rate constant can be determined according to the following equation 2:

$$
k=\frac{I C F_{P} \times[P]}{I C F_{R} \times[R] \times t_{r} \times[A]}
$$


The analyte number density $[A]$ is directly dependant of the sample concentration $(C)$ and instrument parameters such as flow tube temperature $\left(T_{F T}\right.$, in Kelvin) and pressure $\left(P_{F T}\right.$, in $\left.\mathrm{Pa}\right)$, and flow rates of the sample $\left(\Phi_{s}\right)$ and the carrier gas $\left(\Phi_{c}\right)$ (see section 1):

$$
[A]=C \times \frac{\Phi_{S}}{\left(\Phi_{S}+\Phi_{C}\right)} \times \frac{P_{F T}}{k_{B} \times T_{F T}}
$$

with $k_{B}$ the Bolzmann contant $\left(1.380610^{-23} \mathrm{~m}^{2} \mathrm{~kg} \mathrm{~s}^{-2} \mathrm{~K}^{-1}\right)$.

\section{Computational details}

The molecular geometries and reaction energies are obtained by a MP2 optimization using the triple zeta basis set $6-311++\mathrm{g}(\mathrm{d}, \mathrm{p})$. All calculations were carried out using the Gaussian16 program [35]. Geometry optimizations were carried out without any symmetry restrictions in the gas phase.

Collisional rate constants are calculated from parametrized trajectory calculations using the polarizability $\alpha$ and the dipole moment $\mu_{D}$ of the neutral (i.e. the studied molecule) derived from MP2/6-311++g(d,p) calculations and the reduced mass of the ion-molecule complex according to the method described by Su and Chesnavich [36].

\section{Results}

\section{1 - Ion-molecule reactions}

\section{1 - Hydroxide ion $\left(\mathrm{OH}^{-}\right)$reactions}

\subsubsection{With carboxylic acids}

The reaction of carboxylic acids with the $\mathrm{OH}^{-}$precursor ion results in the acidic proton abstraction with product ions at $m / z$ [M-1] [25]:

$$
\mathrm{OH}^{-}+\mathrm{RCOOH} \rightarrow \mathrm{RCOO}^{-}+\mathrm{H}_{2} \mathrm{O}
$$

This work is based on 9 carboxylic acids with MW from $46 \mathrm{~g} \mathrm{~mol}^{-1}$ (formic acid) to $116 \mathrm{~g} \mathrm{~mol}^{-}$ ${ }^{1}$ (caproic and isocaproic acids). Results, summarized in (Table 1), show that proton abstraction is the single reaction pathway between carboxylic acids and $\mathrm{OH}^{-}$. The reaction of $\mathrm{OH}^{-}$with the hydrogen in $\alpha$-position is also possible but thermodynamically less favorable [25] and would also lead to the formation of the deprotonated molecule with a $m / z$ of [M-1].

A competing secondary termolecular reaction can occur between primary ions $[\mathrm{M}-\mathrm{H}]^{-}$and unreacted carboxylic acids molecules (Eq 5) leading to an adduct at $m / z(\mathrm{M}+[\mathrm{M}-1])$ :

$$
\mathrm{RCOOH}+\mathrm{RCOO}^{-}+\mathrm{N}_{2} \rightarrow \mathrm{RCOOH} \cdot \mathrm{RCOO}^{-}+\mathrm{N}_{2}
$$

This analyte cluster was not experimentally observed for butyric, caproic and isocaproic acids.

\subsubsection{With esters}


Esters also react with the $\mathrm{OH}^{-}$precursor to form $[\mathrm{M}-\mathrm{H}]^{-}$ions via a deprotonation according to (Eq 6):

$$
\mathrm{OH}^{-}+\mathrm{R}_{1} \mathrm{COOR}_{2} \rightarrow\left[\mathrm{R}_{1} \mathrm{COOR}_{2}-\mathrm{H}\right]^{-}+\mathrm{H}_{2} \mathrm{O}
$$

Unlike carboxylic acids, this proton abstraction is not the sole reaction pathway between esters and $\mathrm{OH}^{-}$. Fragmentation leading to $\mathrm{R}_{1} \mathrm{COO}^{-}$product anion and a neutral primary alcohol also occurs (Eq 7):

$$
\mathrm{OH}^{-}+\mathrm{R}_{1} \mathrm{COOR}_{2} \rightarrow \mathrm{R}_{1} \mathrm{COO}^{-}+\mathrm{R}_{2} \mathrm{OH}
$$

However, the deprotonation (Eq 6) is the major reaction pathway between esters and $\mathrm{OH}^{-}$, with branching ratios between 65 and $70 \%$.

The reaction is very favorable thermodynamically, especially for the deprotonation on the alpha carbon of the carbonyl. As expected, the negative charge can thus be stabilized by delocalization: for exemple with ethylacetate, an exergonic reaction of $-11.18 \mathrm{kcal} \mathrm{mol}^{-1}$ is evaluated considering the deprotonation of the methyl group, in comparison with -2.08 $\mathrm{kcal} \mathrm{mol}^{-1}$ considering the deprotonation of the first carbon of the ethyl part.

Some data from the literature already describe these two reaction pathways for esters, and in particular for ethyl acetate [26,28]. However, we observe differences in the distribution of these reaction pathways between the literature (deprotonation channel 40\%) and our experimental results (major pathway, 70\%, see Table 1). Note that our experiments were carried out with nitrogen as carrier gas while previous literature results were obtained with helium. It is anticipated that termolecular reactions will be influenced by the change to nitrogen.

We note that the deprotonated molecule (Eq 6) is not experimentally observed with alkyl formate esters. For alkyl formate esters, in addition to fragmentation reaction (Eq 7) another reaction pathway is evidenced and leads to the formation of $\mathrm{R}_{2} \mathrm{O}^{-}$ion together with undetected formic acid (Eq 8). According to calculations and observed branching ratio, both fragmentations of alkyl formates are exergonic but more favorable for Eq $7\left(-44.83 \mathrm{kcal} \mathrm{mol}^{-1} \mathrm{vs}-8.83 \mathrm{kcal} \mathrm{mol}^{-}\right.$ ${ }^{1}$ for ethyl formate).

$$
\mathrm{OH}^{-}+\mathrm{HCOOR}_{2} \rightarrow \mathrm{R}_{2} \mathrm{O}^{-}+\mathrm{HCOOH}
$$

In particular cases, $\mathrm{R}_{2} \mathrm{O}^{-}$and $\mathrm{R}_{1} \mathrm{COO}^{-}$ions can possibly have the same $m / z$, such as ethyl formate leading to $\mathrm{HCOO}^{-}$and $\mathrm{C}_{2} \mathrm{H}_{5} \mathrm{O}^{-}$ions with $\mathrm{m} / z$ of 45 . In such cases, as it is impossible to differentiate these ions, their branching ratios cannot be determined experimentally.

It is important to note that the product ions can associate with neutral molecules, leading to adducts in secondary reactions. Thus, $\mathrm{R}_{2} \mathrm{O}^{-}$ion from reaction $(\mathrm{Eq} 8)$ and neutral $\mathrm{R}_{2} \mathrm{OH}$ from reaction (Eq 7) react together and form $\mathrm{R}_{2} \mathrm{OH} . \mathrm{R}_{2} \mathrm{O}^{-}$as secondary product ion. Neutral molecules of water and carbon dioxide from the matrix also act as reagents in secondary reactions with product ions. Experimentally, we notice the formation of $\mathrm{CO}_{2} \cdot[\mathrm{M}-\mathrm{H}]^{-}, \mathrm{H}_{2} \mathrm{O} \cdot \mathrm{R}_{2} \mathrm{O}^{-}$and $\mathrm{CO}_{2} \cdot \mathrm{R}_{2} \mathrm{O}^{-}$ when studying esters in synthetic air matrices containing water and carbon dioxide. The formation of these cluster ions is directly linked to the quantity of water and $\mathrm{CO}_{2}$ in the flow tube (i.e. their concentration in the sample matrix). These secondary ions have absolutely to be considered in order to limit as much as possible the influence of the matrix on the SIFT-MS quantification.

We must also highlight that in the presence of the hydroxide ion and water in the flow tube, hydrated hydroxide ions $\mathrm{OH}^{-} \cdot \mathrm{H}_{2} \mathrm{O}$ and $\mathrm{OH}^{-} .\left(\mathrm{H}_{2} \mathrm{O}\right)_{2}$ are formed. Various studies documented the 
role of these hydrated hydroxide ions in the reactivity of the analytes in SIFT-MS [28,32]. Thus, these ions also act as precursors and can also lead to the formation of the $\mathrm{RCOO}^{-}$product ions according to Eq 9 (carboxylic acids) and Eq 10 (esters):

$$
\begin{aligned}
& \mathrm{OH}^{-} .\left(\mathrm{H}_{2} \mathrm{O}\right)_{n}+\mathrm{RCOOH} \rightarrow \mathrm{RCOO}^{-}+(n+1) \mathrm{H}_{2} \mathrm{O} \\
& \mathrm{OH}^{-} .\left(\mathrm{H}_{2} \mathrm{O}\right)_{n}+\mathrm{R}_{1} \mathrm{COOR}_{2} \rightarrow \mathrm{R}_{1} \mathrm{COO}^{-}+\mathrm{R}_{2} \mathrm{OH}+n \mathrm{H}_{2} \mathrm{O}
\end{aligned}
$$

\section{2 - Superoxide ion $\left(\mathrm{O}_{2}^{\bullet-}\right)$}

\subsubsection{With carboxylic acids}

The reaction between superoxide ion and carboxylic acids leads to deprotonation (Eq 11):

$$
\mathrm{O}_{2}^{\bullet-}+\mathrm{RCOOH} \rightarrow \mathrm{RCOO}^{-}+\mathrm{HOO}^{\bullet}
$$

Indeed, this deprotonation is not the only possible reaction way with the $\mathrm{O}_{2}{ }^{\bullet-}$ ion, in particular for the heavier acids. Thus with valeric, isovaleric, caproic and isocaproic acids, association reactions are observed leading to ${\mathrm{M} . \mathrm{O}_{2}}^{\bullet-}$ ions with $\mathrm{m} / \mathrm{z}$ of 134 (valeric and isovaleric) and 148 (caproic and isocaproic) (Eq 12):

$$
\mathrm{O}_{2}{ }^{\bullet-}+\mathrm{RCOOH}+\mathrm{N}_{2} \rightarrow \mathrm{RCOOH} \mathrm{O}_{2}{ }^{\bullet-}+\mathrm{N}_{2}
$$

This latter reaction is not predominant with branching ratios ranging from 6\% (isovaleric acid) to $20 \%$ (caproic and isocaproic) (Table 1).

\subsubsection{With esters}

Esters and $\mathrm{O}_{2}{ }^{\bullet-}$ precursor ion react according to the following reaction:

$$
\mathrm{O}_{2}^{\bullet-}+\mathrm{R}_{1} \mathrm{COOR}_{2} \rightarrow \mathrm{R}_{1} \mathrm{COO}^{-}+\mathrm{R}_{2}^{\bullet}+\mathrm{O}_{2}
$$

Experimentally, $\mathrm{R}_{1} \mathrm{COO}^{-}$ions are detected but only for a few esters (see Table 1) and with very low signal intensity meaning that reaction 13 seems to be possible but not very favorable. Calculation results indicate that this reaction is slightly more favorable with alkyl formate esters $\left(-11 \mathrm{kcal} \mathrm{mol}^{-1}\right)$ than with the others esters (between $\sim-7 \mathrm{kcal} \mathrm{mol}^{-1}$ and $-3 \mathrm{kcal} \mathrm{mol}^{-1}$ for butyl propionate and ethyl butyrate).

\section{$1.3-\mathrm{O}^{\bullet-}$ radical ion reactions}

\subsubsection{With carboxylic acids}

Experimentally, the reaction between carboxylic acids and the $\mathrm{O}^{\bullet-}$ radical ion leads to the formation of the deprotonated molecules at $m / z$ [M-1] according to the following reaction (Eq 14):

$$
\mathrm{O}^{\bullet-}+\mathrm{RCOOH} \rightarrow \mathrm{RCOO}^{-}+\mathrm{HO}^{\bullet}
$$

Reactions between these $\mathrm{RCOO}^{-}$product ions and unreacted carboxylic acids can occur in the flow tube to form $\mathrm{RCOOH}$. $\mathrm{RCOO}^{-}$adducts. 
In addition to reaction (Eq 14), the following reaction can also be considered (Eq 15):

$$
\mathrm{O}^{\bullet-}+\mathrm{RCOOH} \rightarrow \mathrm{RCOO}^{\bullet}+\mathrm{HO}^{-}
$$

The uncharged $\mathrm{RCOO}^{\bullet}$ radical cannot be observed in SIFT-MS. The product ion to follow here is therefore $\mathrm{OH}^{-}$. However, the $\mathrm{OH}^{-}$ion $(\mathrm{m} / z, 17)$ is already observed in the flow tube in the absence of analyte [32]. Although an increase in the signal at $\mathrm{m} / \mathrm{z}, 17$ is to be noted in the presence of each carboxylic acid, it is difficult to conclude that the formation of additional $\mathrm{OH}^{-}$ ions results from reaction 15 between carboxylic acids and $\mathrm{O}^{\bullet-}$ radical ion. Numerical simulations do not explain this observation especially since the thermodynamic calculation is unfavorable except for formic acid.

\subsubsection{With esters}

Esters also react with $\mathrm{O}^{\bullet-}$ radical ion to form a deprotonated molecule (Eq 16) but, as noticed with the hydroxide ion, this reaction is not observed for alkyl formate esters.

$$
\mathrm{O}^{\bullet-}+\mathrm{R}_{1} \mathrm{COOR}_{2} \rightarrow\left[\mathrm{R}_{1} \mathrm{COOR}_{2}-\mathrm{H}\right]^{-}+\mathrm{HO}^{\bullet}
$$

In the same way as for the carboxylic acids, the formation of the uncharged $\left[\mathrm{R}_{1} \mathrm{COOR}_{2}-\mathrm{H}\right]^{\bullet}$ cannot be excluded since it cannot be observed in SIFT-MS.

The formation of $\mathrm{R}_{1} \mathrm{COO}^{-}$is also observed with $\mathrm{O}^{\bullet-}$ precursor ion (Eq 17). This second reaction is the major pathway of the reaction between esters and $\mathrm{O}^{\bullet-}$ ion with branching ratios between 48 and $85 \%$ (Table 1):

$$
\mathrm{O}^{\bullet-}+\mathrm{R}_{1} \mathrm{COOR}_{2} \rightarrow \mathrm{R}_{1} \mathrm{COO}^{-}+\mathrm{R}_{2} \mathrm{O}^{\bullet}
$$

and:

$$
\mathrm{O}^{\bullet-}+\mathrm{R}_{1} \mathrm{COOR}_{2} \rightarrow \mathrm{R}_{1} \mathrm{COO}^{\bullet}+\mathrm{R}_{2} \mathrm{O}^{-}
$$

There are two channels to the reaction between $\mathrm{O}^{\bullet-}$ and $\mathrm{R}_{1} \mathrm{COOR}_{2}(\mathrm{Eq} 17$ and 18). Secondary reactions of $\mathrm{R}_{2} \mathrm{O}^{-}$with $\mathrm{CO}_{2}, \mathrm{H}_{2} \mathrm{O}$ and $\mathrm{R}_{2} \mathrm{OH}$ are also observed. $\mathrm{R}_{2} \mathrm{OH}^{-} \cdot \mathrm{H}_{2} \mathrm{O}$ and $\mathrm{R}_{2} \mathrm{O}^{-} \cdot \mathrm{R}_{2} \mathrm{OH}$ ions are systematically observed from the alkyl formate esters but never for the other studied esters. 
Table 1. Product ions for reaction of carboxylic acids and esters with negative precursor ions, at $393 \mathrm{~K}$, in $\mathrm{N}_{2}$.

\begin{tabular}{|c|c|c|c|c|c|c|c|c|c|}
\hline \multirow[b]{2}{*}{$\begin{array}{l}\text { Compounds } \\
\text { Formula }\end{array}$} & \multirow{2}{*}{$\begin{array}{c}\text { MW } \\
\text { Purity }(\%) \\
\text { CAS } \\
\text { number }\end{array}$} & \multicolumn{2}{|l|}{$\mathbf{O H}^{-}$} & \multicolumn{2}{|l|}{$\mathbf{O}_{2}{ }^{--}$} & \multicolumn{2}{|l|}{$\mathbf{O}^{*-}$} & \multicolumn{2}{|l|}{$\mathrm{NO}_{2}^{-}$} \\
\hline & & Product ions $(\mathrm{m} / \mathrm{z})$ & BR & Product ions $(m / z)$ & BR & Product ions $(\mathrm{m} / \mathrm{z})$ & BR & Product ions $(\mathrm{m} / \mathrm{z})$ & $\mathrm{BR}$ \\
\hline \multicolumn{10}{|c|}{ Carboxylic acids } \\
\hline $\begin{array}{l}\text { Formic acid } \\
\mathrm{HCOOH}\end{array}$ & $\begin{array}{c}\mathbf{4 6} \\
\geq 95.0 \\
64-18-6\end{array}$ & $\begin{array}{l}\text { HCOO- }^{-}(45) \\
\text { M.HCOO }^{-}(91)\end{array}$ & 1.0 & $\begin{array}{l}\operatorname{HCOO}^{-}(45) \\
\text { M.HCOO }^{-}(91)\end{array}$ & 1.0 & $\begin{array}{l}\text { HCOO- }^{-}(45) \\
\text { M.HCOO }^{-}(91)\end{array}$ & 1.0 & $\begin{array}{l}\mathrm{HCOO}^{-}(45) \\
{\mathrm{M} . \mathrm{NO}_{2}^{-}}^{-}(92)\end{array}$ & $\begin{array}{l}0.36 \\
0.64\end{array}$ \\
\hline $\begin{array}{l}\text { Acetic acid } \\
\mathrm{CH}_{3} \mathrm{COOH}\end{array}$ & $\begin{array}{c}60 \\
\geq 99.0 \\
64-19-7\end{array}$ & $\begin{array}{c}\mathrm{CH}_{3} \mathrm{COO}^{-}(59) \\
M_{.} \mathrm{CH}_{3} \mathrm{COO}^{-}(119)\end{array}$ & 1.0 & $\begin{array}{c}\mathrm{CH}_{3} \mathrm{COO}^{-}(59) \\
\mathrm{M.CH}_{3} \mathrm{COO}^{-}(119)\end{array}$ & 1.0 & $\begin{array}{c}\mathrm{CH}_{3} \mathrm{COO}^{-}(59) \\
M_{3} \mathrm{CH}_{3} \mathrm{COO}^{-}(119)\end{array}$ & 1.0 & $\begin{array}{l}\mathrm{CH}_{3} \mathrm{COO}^{-}(59) \\
\mathrm{M}^{-N_{2}}{ }_{2}^{-}(106)\end{array}$ & $\begin{array}{l}0.22 \\
0.78\end{array}$ \\
\hline $\begin{array}{l}\text { Propionic acid } \\
\mathrm{C}_{2} \mathrm{H}_{5} \mathrm{COOH}\end{array}$ & $\begin{array}{c}74 \\
\geq 99.5 \\
79-09-4\end{array}$ & $\begin{array}{c}\mathrm{C}_{2} \mathrm{H}_{5} \mathrm{COO}^{-}(73) \\
M . \mathrm{C}_{2} \mathrm{H}_{5} \mathrm{COO}^{-}(147)\end{array}$ & 1.0 & $\begin{array}{c}\mathrm{C}_{2} \mathrm{H}_{5} \mathrm{COO}^{-}(73) \\
M . \mathrm{C}_{2} \mathrm{H}_{5} \mathrm{COO}^{-}(147)\end{array}$ & 1.0 & $\begin{array}{c}\mathrm{C}_{2} \mathrm{H}_{5} \mathrm{COO}^{-}(73) \\
M . \mathrm{C}_{2} \mathrm{H}_{5} \mathrm{COO}^{-}(147)\end{array}$ & 1.0 & $\begin{array}{c}\mathrm{C}_{2} \mathrm{H}_{5} \mathrm{COO}^{-}(73) \\
\mathrm{M}^{-N_{2}^{-}}(120)\end{array}$ & $\begin{array}{l}0.03 \\
0.97\end{array}$ \\
\hline $\begin{array}{l}\text { Butyric acid } \\
\mathrm{C}_{3} \mathrm{H}_{7} \mathrm{COOH}\end{array}$ & $\begin{array}{c}88 \\
\geq 99.0 \\
107-92-6\end{array}$ & $\mathrm{C}_{3} \mathrm{H}_{7} \mathrm{COO}^{-}(87)$ & 1.0 & $\mathrm{C}_{3} \mathrm{H}_{7} \mathrm{COO}^{-}(87)$ & 1.0 & $\mathrm{C}_{3} \mathrm{H}_{7} \mathrm{COO}^{-}(87)$ & 1.0 & $\begin{array}{l}\mathrm{C}_{3} \mathrm{H}_{7} \mathrm{COO}^{-}(87) \\
\text { M. } \mathrm{NO}_{2}^{-}(134)\end{array}$ & $\begin{array}{l}0.13 \\
0.87\end{array}$ \\
\hline $\begin{array}{l}\text { Isobutyric acid } \\
\left(\mathrm{CH}_{3}\right)_{2} \mathrm{CHCOOH}\end{array}$ & $\begin{array}{c}88 \\
\geq 99.0 \\
79-31-2 \\
\end{array}$ & 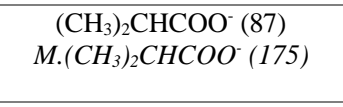 & 1.0 & $\begin{array}{c}\left(\mathrm{CH}_{3}\right)_{2} \mathrm{CHCOO}^{-}(87) \\
M .\left(\mathrm{CH}_{3}\right)_{2} \mathrm{CHCOO}^{-}(175)\end{array}$ & 1.0 & $\begin{array}{c}\left(\mathrm{CH}_{3}\right)_{2} \mathrm{CHCOO}^{-}(87) \\
M_{(}\left(\mathrm{CH}_{3}\right)_{2} \mathrm{CHCOO}^{-}(175)\end{array}$ & 1.0 & $\begin{array}{c}\left(\mathrm{CH}_{3}\right)_{2} \mathrm{CHCOO}^{-}(87) \\
\mathrm{M} . \mathrm{NO}_{2}^{-}(134)\end{array}$ & $\begin{array}{l}0.03 \\
0.97\end{array}$ \\
\hline $\begin{array}{l}\text { Valeric acid } \\
\mathrm{C}_{4} \mathrm{H}_{9} \mathrm{COOH}\end{array}$ & $\begin{array}{c}102 \\
\geq 99.8 \\
109-52-4\end{array}$ & $\begin{array}{c}\mathrm{C}_{4} \mathrm{H}_{9} \mathrm{COO}^{-}(101) \\
M_{.} \mathrm{C}_{4} \mathrm{H}_{9} \mathrm{COO}^{-}(203)\end{array}$ & 1.0 & $\begin{array}{c}\mathrm{C}_{4} \mathrm{H}_{9} \mathrm{COO}^{-}(101) \\
M . \mathrm{C}_{4} \mathrm{H}_{9} \mathrm{COO}^{-}(203) \\
\mathrm{M.O}_{2}^{-}(134)\end{array}$ & $\begin{array}{l}0.9 \\
0.1\end{array}$ & $\begin{array}{c}\mathrm{C}_{4} \mathrm{H}_{9} \mathrm{COO}^{-}(101) \\
M_{.} \mathrm{C}_{4} \mathrm{H}_{9} \mathrm{COO}^{-}(203)\end{array}$ & 1.0 & $\begin{array}{c}\mathrm{C}_{4} \mathrm{H}_{9} \mathrm{COO}^{-}(101) \\
\mathrm{M}_{.} \mathrm{NO}_{2}^{-}(148)\end{array}$ & $\begin{array}{l}0.01 \\
0.99\end{array}$ \\
\hline $\begin{array}{c}\text { Isovaleric acid } \\
\left(\mathrm{CH}_{3}\right)_{2} \mathrm{CHCH}_{2} \mathrm{COOH}\end{array}$ & $\begin{array}{c}102 \\
\geq 98.5 \\
503-74-2\end{array}$ & $\begin{array}{c}\left(\mathrm{CH}_{3}\right)_{2} \mathrm{CHCH}_{2} \mathrm{COO}^{-}(101) \\
M .\left(\mathrm{CH}_{3}\right)_{2} \mathrm{CHCH}_{2} \mathrm{COO}^{-} \\
(203)\end{array}$ & 1.0 & $\begin{array}{c}\left(\mathrm{CH}_{3}\right)_{2} \mathrm{CHCH}_{2} \mathrm{COO}^{-}(101) \\
\text { M. } \mathrm{C}_{4} \mathrm{H}_{9} \mathrm{COO}^{-}(203) \\
{\mathrm{M} . \mathrm{O}_{2}^{-}}^{-}(134)\end{array}$ & 0.94 & $\begin{array}{c}\left(\mathrm{CH}_{3}\right)_{2} \mathrm{CHCH}_{2} \mathrm{COO}^{-}(101) \\
M .\left(\mathrm{CH}_{3}\right)_{2} \mathrm{CHCH}_{2} \mathrm{COO}^{-} \\
(2 \mathrm{O})\end{array}$ & 1.0 & $\begin{array}{c}\left(\mathrm{CH}_{3}\right)_{2} \mathrm{CHCH}_{2} \mathrm{COO}^{-}(101) \\
{\mathrm{M} . \mathrm{NO}_{2}^{-}}^{-}(148)\end{array}$ & $\begin{array}{l}0.02 \\
0.98\end{array}$ \\
\hline $\begin{array}{l}\text { Caproic acid } \\
\mathrm{C}_{5} \mathrm{H}_{11} \mathrm{COOH}\end{array}$ & $\begin{array}{c}116 \\
\geq 99.0 \\
142-62-1\end{array}$ & $\mathrm{C}_{5} \mathrm{H}_{11} \mathrm{COO}^{-}(115)$ & 1.0 & $\begin{array}{l}\mathrm{C}_{5} \mathrm{H}_{11} \mathrm{COO}^{-}(115) \\
\quad \mathrm{M}^{-} \mathrm{O}_{2}^{-}(148)\end{array}$ & $\begin{array}{l}0.8 \\
0.2\end{array}$ & $\mathrm{C}_{5} \mathrm{H}_{11} \mathrm{COO}^{-}(115)$ & 1.0 & $\begin{array}{l}\mathrm{C}_{5} \mathrm{H}_{11} \mathrm{COO}^{-}(115) \\
{\mathrm{M} . \mathrm{NO}_{2}^{-}}^{-}(162)\end{array}$ & $\begin{array}{l}0.03 \\
0.97\end{array}$ \\
\hline $\begin{array}{c}\text { Isocaproic acid } \\
\left(\mathrm{CH}_{3}\right)_{2} \mathrm{CH}\left(\mathrm{CH}_{2}\right)_{2} \mathrm{COOH}\end{array}$ & $\begin{array}{c}116 \\
99.0 \\
646-07-1 \\
\end{array}$ & $\left(\mathrm{CH}_{3}\right)_{2} \mathrm{CH}\left(\mathrm{CH}_{2}\right)_{2} \mathrm{COO}^{-}(115)$ & 1.0 & $\begin{array}{c}\left(\mathrm{CH}_{3}\right)_{2} \mathrm{CH}_{(}\left(\mathrm{CH}_{2}\right)_{2} \mathrm{COO}^{-} \\
(115) \\
{\mathrm{M} . \mathrm{O}_{2}^{-}}^{-}(148) \\
\text { Esters }\end{array}$ & $\begin{array}{l}0.8 \\
0.2\end{array}$ & $\left(\mathrm{CH}_{3}\right)_{2} \mathrm{CH}\left(\mathrm{CH}_{2}\right)_{2} \mathrm{COO}^{-}(115)$ & 1.0 & $\begin{array}{c}\left(\mathrm{CH}_{3}\right)_{2} \mathrm{CH}\left(\mathrm{CH}_{2}\right)_{2} \mathrm{COO}^{-} \\
(115) \\
\text { M.NO } \\
{ }^{-}(162)\end{array}$ & $\begin{array}{l}0.01 \\
0.99\end{array}$ \\
\hline $\begin{array}{l}\text { Methyl formate } \\
\mathrm{HCOOCH}_{3}\end{array}$ & $\begin{array}{c}60 \\
\geq 99.8 \\
107-31-3\end{array}$ & $\begin{array}{c}\mathrm{HCOO}^{-}(45) \\
\mathrm{CH}_{3} \mathrm{O}^{-}(31) \\
\mathrm{CH}_{3} \mathrm{O} \cdot \mathrm{H}_{2} \mathrm{O}(49) \\
\mathrm{CH}_{3} \mathrm{O} \cdot \mathrm{CO}_{2}(75) \\
\mathrm{CH}_{3} \mathrm{O} \cdot \mathrm{CH}_{3} \mathrm{OH}(63) \\
\end{array}$ & $\begin{array}{l}0.35 \\
0.65\end{array}$ & $\begin{array}{c}\text { Esters } \\
\mathrm{HCOO}^{-}(45)\end{array}$ & 1.0 & $\begin{array}{c}\mathrm{HCOO}^{-}(45) \\
\mathrm{CH}_{3} \mathrm{O}^{-}(31) \\
\mathrm{CH}_{3} \mathrm{O} \cdot \mathrm{H}_{2} \mathrm{O}(49) \\
\mathrm{CH}_{3} \mathrm{O} \cdot \mathrm{CO}_{2}(49) \\
\mathrm{CH}_{3} \mathrm{O} \cdot \mathrm{CH}_{3} \mathrm{OH}(63)\end{array}$ & $\begin{array}{l}0.67 \\
0.33\end{array}$ & & \\
\hline $\begin{array}{l}\text { Methyl acetate } \\
\mathrm{CH}_{3} \mathrm{COOCH}_{3}\end{array}$ & $\begin{array}{c}74 \\
\geq 99.9 \\
79-20-9\end{array}$ & $\begin{array}{c}\mathrm{CH}_{3} \mathrm{COO}^{-}(59) \\
\mathrm{M}-1(73)\end{array}$ & $\begin{array}{l}0.08 \\
0.92\end{array}$ & & & $\begin{array}{c}\mathrm{CH}_{3} \mathrm{COO}^{-}(59) \\
\mathrm{M}-1(73) \\
\mathrm{CH}_{3} \mathrm{O}^{-}(31) \\
\mathrm{CH}_{3} \mathrm{O}^{-} . \mathrm{CO}_{2}(75)\end{array}$ & $\begin{array}{l}0.48 \\
0.48 \\
0.04\end{array}$ & & \\
\hline Methyl propionate & 88 & $\mathrm{C}_{2} \mathrm{H}_{5} \mathrm{COO}^{-}(73)$ & 0.22 & & & $\mathrm{C}_{2} \mathrm{H}_{5} \mathrm{COO}^{-}(73)$ & 0.55 & & \\
\hline
\end{tabular}




\begin{tabular}{|c|c|c|c|c|c|c|c|}
\hline $\mathrm{C}_{2} \mathrm{H}_{5} \mathrm{COOCH}_{3}$ & $\begin{array}{c}\geq 99.8 \\
554-12-1\end{array}$ & $\begin{array}{c}\mathrm{M}-1(87) \\
M-1 . \mathrm{CO}_{2}(131)\end{array}$ & 0.78 & & & $\begin{array}{c}\mathrm{M}-1(87) \\
M-1 . \mathrm{CO}_{2}(131) \\
\mathrm{CH}_{3} \mathrm{O}^{-}(31) \\
\mathrm{CH}_{3} \mathrm{O}^{-} . \mathrm{CO}_{2}(75)\end{array}$ & $\begin{array}{l}0.25 \\
0.20\end{array}$ \\
\hline Methyl butyrate & 102 & $\mathrm{C}_{3} \mathrm{H}_{7} \mathrm{COO}^{-}(87)$ & 0.16 & & & $\mathrm{C}_{3} \mathrm{H}_{7} \mathrm{COO}^{-}(87)$ & 0.57 \\
\hline $\mathrm{C}_{3} \mathrm{H}_{7} \mathrm{COOCH}_{3}$ & $\begin{array}{c}\geq 99.5 \\
623-42-7\end{array}$ & $\begin{array}{c}\mathrm{M}-1(101) \\
M-1 . \mathrm{CO}_{2}(145)\end{array}$ & 0.84 & & & $\begin{array}{c}\text { M-1 (101) } \\
M-1 . C O_{2}(145)\end{array}$ & 0.19 \\
\hline & & & & & & 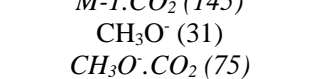 & 0.24 \\
\hline Methyl valerate & 116 & $\mathrm{C}_{4} \mathrm{H}_{9} \mathrm{COO}^{-}(101)$ & 0.11 & $\mathrm{C}_{4} \mathrm{H}_{9} \mathrm{COO}^{-}(101)$ & 1.0 & $\mathrm{C}_{4} \mathrm{H}_{9} \mathrm{COO}^{-}(101)$ & 0.55 \\
\hline $\mathrm{C}_{4} \mathrm{H}_{9} \mathrm{COOCH}_{3}$ & $\begin{array}{c}\geq 99.8 \\
624-24-8\end{array}$ & M-1 (115) & 0.89 & & & $\begin{array}{c}\mathrm{M}-1(115) \\
\mathrm{CH}_{3} \mathrm{O}^{-}(31) \\
\mathrm{CH}_{3} \mathrm{O}^{-} \cdot \mathrm{CO}_{2}(75)\end{array}$ & $\begin{array}{l}0.23 \\
0.22\end{array}$ \\
\hline $\begin{array}{l}\text { Methyl caproate } \\
\mathrm{C}_{5} \mathrm{H}_{11} \mathrm{COOCH}_{3}\end{array}$ & $\begin{array}{c}130 \\
\geq 99.8 \\
106-70-7\end{array}$ & $\begin{array}{l}\mathrm{C}_{5} \mathrm{H}_{11} \mathrm{COO}^{-}(115) \\
\mathrm{M}-1(129)\end{array}$ & $\begin{array}{l}0.13 \\
0.87\end{array}$ & & & $\begin{array}{c}\mathrm{C}_{5} \mathrm{H}_{11} \mathrm{COO}^{-}(115) \\
\mathrm{M}-1(129) \\
\mathrm{CH}_{3} \mathrm{O}^{-}(31) \\
\mathrm{CH}_{3} \mathrm{O}^{-} \cdot \mathrm{CO}_{2}(75) \\
\end{array}$ & $\begin{array}{l}0.59 \\
0.15 \\
0.26\end{array}$ \\
\hline $\begin{array}{l}\text { Ethyl formate } \\
\mathrm{HCOOC}_{2} \mathrm{H}_{5}\end{array}$ & $\begin{array}{c}74 \\
\geq 99.5 \\
109-94-4\end{array}$ & $\begin{array}{c}\mathrm{HCOO}^{-}(45) \\
\mathrm{C}_{2} \mathrm{H}_{5} \mathrm{O}^{-}(45) \\
C_{2} \mathrm{H}_{5} \mathrm{O}^{-} \cdot \mathrm{H}_{2} \mathrm{O}(63) \\
\mathrm{C}_{2} \mathrm{H}_{5} \mathrm{O} \cdot \mathrm{CO} \mathrm{O}_{2}(89) \\
\mathrm{C}_{2} \mathrm{H}_{5} \mathrm{O}^{-} \cdot \mathrm{C}_{2} \mathrm{H}_{5} \mathrm{OH}(91) \\
\end{array}$ & $\begin{array}{l}\text { nd } \\
\text { nd }\end{array}$ & $\mathrm{HCOO}^{-}(45)$ & 1.0 & $\begin{array}{c}\mathrm{HCOO}^{-}(45) \\
\mathrm{C}_{2} \mathrm{H}_{5} \mathrm{O}^{-}(45) \\
C_{2} H_{5} O \cdot H_{2} O(63) \\
C_{2} H_{5} O \cdot C O_{2}(89) \\
C_{2} H_{5} O \cdot C_{2} H_{5} O H(91) \\
\end{array}$ & $\begin{array}{l}\text { nd } \\
\text { nd }\end{array}$ \\
\hline $\begin{array}{l}\text { Ethyl acetate } \\
\mathrm{CH}_{3} \mathrm{COOC}_{2} \mathrm{H}_{5}\end{array}$ & $\begin{array}{c}88 \\
\geq 99.9 \\
141-78-6\end{array}$ & $\begin{array}{c}\mathrm{CH}_{3} \mathrm{COO}^{-}(59) \\
\mathrm{M}-1(87) \\
M-1 . \mathrm{CO}_{2}(131) \\
\end{array}$ & $\begin{array}{l}0.30 \\
0.70\end{array}$ & & & $\begin{array}{c}\mathrm{CH}_{3} \mathrm{COO}^{-}(59) \\
\mathrm{M}-1(87) \\
\mathrm{C}_{2} \mathrm{H}_{5} \mathrm{O}^{-}(45) \\
\mathrm{C}_{2} \mathrm{H}_{5} \mathrm{O}^{-} \cdot \mathrm{CO}_{2}(89) \\
\end{array}$ & $\begin{array}{l}0.81 \\
0.12 \\
0.07\end{array}$ \\
\hline $\begin{array}{l}\text { Ethyl butyrate } \\
\mathrm{C}_{3} \mathrm{H}_{7} \mathrm{COOC}_{2} \mathrm{H}_{5}\end{array}$ & $\begin{array}{c}116 \\
\geq 99.5 \\
105-54-4\end{array}$ & $\begin{array}{c}\mathrm{C}_{3} \mathrm{H}_{7} \mathrm{COO}^{-}(87) \\
\mathrm{M}-1(115)\end{array}$ & $\begin{array}{l}0.28 \\
0.72\end{array}$ & $\mathrm{C}_{3} \mathrm{H}_{7} \mathrm{COO}^{-}(87)$ & 1.0 & $\begin{array}{c}\mathrm{C}_{3} \mathrm{H}_{7} \mathrm{COO}^{-}(87) \\
\mathrm{M}-1(115) \\
\mathrm{C}_{2} \mathrm{H}_{5} \mathrm{O}^{-}(45) \\
C_{2} \mathrm{H}_{5} \mathrm{O}^{-} \cdot \mathrm{CO}_{2}(89) \\
\end{array}$ & $\begin{array}{l}0.79 \\
0.08 \\
0.13\end{array}$ \\
\hline $\begin{array}{l}\text { Ethyl valerate } \\
\mathrm{C}_{4} \mathrm{H}_{9} \mathrm{COOC}_{2} \mathrm{H}_{5}\end{array}$ & $\begin{array}{c}130 \\
\geq 98.0 \\
539-82-2\end{array}$ & $\begin{array}{l}\mathrm{C}_{4} \mathrm{H}_{9} \mathrm{COO}^{-}(101) \\
\mathrm{M}-1(129)\end{array}$ & $\begin{array}{l}0.28 \\
0.72\end{array}$ & & & $\begin{array}{c}\mathrm{C}_{4} \mathrm{H}_{9} \mathrm{COO}^{-}(101) \\
\mathrm{M}-1(129) \\
\mathrm{C}_{2} \mathrm{H}_{5} \mathrm{O}^{-}(45) \\
\mathrm{C}_{2} \mathrm{H}_{5} \mathrm{O}^{-} \cdot \mathrm{CO}_{2}(89) \\
\end{array}$ & $\begin{array}{l}0.85 \\
0.10 \\
0.05\end{array}$ \\
\hline $\begin{array}{l}\text { Ethyl 3-methylbutanoate } \\
\left(\mathrm{CH}_{3}\right)_{2} \mathrm{CHCH}_{2} \mathrm{COOC}_{2} \mathrm{H}_{5}\end{array}$ & $\begin{array}{c}130 \\
98.0 \\
108-64-5\end{array}$ & $\begin{array}{c}\left(\mathrm{CH}_{3}\right)_{2} \mathrm{CHCH}_{2} \mathrm{COO}^{-}(101) \\
\mathrm{M}-1(129)\end{array}$ & $\begin{array}{l}0.28 \\
0.72\end{array}$ & $\left(\mathrm{CH}_{3}\right)_{2} \mathrm{CHCH}_{2} \mathrm{COO}^{-}(101)$ & 1.0 & $\begin{array}{c}\left(\mathrm{CH}_{3}\right)_{2} \mathrm{CHCH}_{2} \mathrm{COO}^{-}(101) \\
\mathrm{M}-1(129) \\
\mathrm{C}_{2} \mathrm{H}_{5} \mathrm{O}^{-}(45) \\
\mathrm{C}_{2} \mathrm{H}_{5} \mathrm{O}^{-} \cdot \mathrm{CO}_{2}(89) \\
\end{array}$ & $\begin{array}{l}0.85 \\
0.10 \\
0.05\end{array}$ \\
\hline $\begin{array}{l}\text { Ethyl caproate } \\
\mathrm{C}_{5} \mathrm{H}_{11} \mathrm{COOC}_{2} \mathrm{H}_{5}\end{array}$ & $\begin{array}{c}144 \\
\geq 99.0 \\
123-66-0\end{array}$ & $\begin{array}{c}\mathrm{C}_{5} \mathrm{H}_{11} \mathrm{COO}^{-}(115) \\
\mathrm{M}-1(143)\end{array}$ & $\begin{array}{l}0.24 \\
0.76\end{array}$ & $\mathrm{C}_{5} \mathrm{H}_{11} \mathrm{COO}^{-}(115)$ & 1.0 & $\begin{array}{c}\mathrm{C}_{5} \mathrm{H}_{11} \mathrm{COO}^{-}(115) \\
\mathrm{M}-1(143) \\
\mathrm{C}_{2} \mathrm{H}_{5} \mathrm{O}^{-}(45) \\
\mathrm{C}_{2} \mathrm{H}_{5} \mathrm{O}^{-} \cdot \mathrm{CO}_{2}(89) \\
\end{array}$ & $\begin{array}{l}0.79 \\
0.06 \\
0.15\end{array}$ \\
\hline $\begin{array}{l}\text { Propyl formate } \\
\mathrm{HCOOC}_{3} \mathrm{H}_{7}\end{array}$ & $\begin{array}{c}88 \\
97.0 \\
110-74-7\end{array}$ & $\begin{array}{c}\mathrm{HCOO}^{-}(45) \\
\mathrm{C}_{3} \mathrm{H}_{7} \mathrm{O}^{-}(59) \\
C_{3} H_{7} O^{-} \cdot H_{2} O(77) \\
C_{3} H_{7} O \cdot \mathrm{O}_{2}(103) \\
C_{3} H_{7} O_{2} \cdot C_{3} H_{7} O H(119) \\
\end{array}$ & $\begin{array}{l}0.85 \\
0.15\end{array}$ & $\mathrm{HCOO}^{-}(45)$ & 1.0 & $\begin{array}{c}\mathrm{HCOO}^{-}(45) \\
\mathrm{C}_{3} \mathrm{H}_{7} \mathrm{O}^{-}(59) \\
C_{3} H_{7} O \cdot \mathrm{H}_{2} O(77) \\
C_{3} \mathrm{H}_{7} O \cdot \mathrm{CO}_{2}(103) \\
C_{3} \mathrm{H}_{7} O \cdot \mathrm{C}_{3} \mathrm{H}_{7} \mathrm{OH}(119) \\
\end{array}$ & $\begin{array}{l}0.82 \\
0.18\end{array}$ \\
\hline Propyl acetate & 102 & $\mathrm{CH}_{3} \mathrm{COO}^{-}(59)$ & 0.25 & & & $\mathrm{CH}_{3} \mathrm{COO}^{-}(59)$ & nd \\
\hline
\end{tabular}




\begin{tabular}{|c|c|c|c|c|c|c|c|}
\hline $\mathrm{CH}_{3} \mathrm{COOC}_{3} \mathrm{H}_{7}$ & $\begin{array}{c}\geq 99.7 \\
109-60-4\end{array}$ & $\begin{array}{c}\mathrm{M}-1(101) \\
M-1 . C O_{2}(145)\end{array}$ & 0.75 & & & $\begin{array}{c}\mathrm{M}-1(101) \\
\mathrm{C}_{3} \mathrm{H}_{7} \mathrm{O}^{-}(59) \\
\mathrm{C}_{3} \mathrm{H}_{7} \mathrm{O}^{-} \cdot \mathrm{CO}_{2}(103)\end{array}$ & $\begin{array}{c}0.17 \\
\text { nd }\end{array}$ \\
\hline \multirow{4}{*}{$\begin{array}{c}\text { Propyl propionate } \\
\mathrm{C}_{2} \mathrm{H}_{5} \mathrm{COOC}_{3} \mathrm{H}_{7}\end{array}$} & 116 & $\mathrm{C}_{2} \mathrm{H}_{5} \mathrm{COO}^{-}(73)$ & 0.24 & $\mathrm{C}_{2} \mathrm{H}_{5} \mathrm{COO}^{-}(73)$ & 1.0 & $\mathrm{C}_{2} \mathrm{H}_{5} \mathrm{COO}^{-}(73)$ & 0.80 \\
\hline & 99.0 & M-1 (115) & 0.76 & & & M-1 (115) & 0.09 \\
\hline & $106-36-5$ & $\mathrm{M}-1 . \mathrm{CO}_{2}(159)$ & & & & $\mathrm{C}_{3} \mathrm{H}_{7} \mathrm{O}^{-}(59)$ & 0.11 \\
\hline & & & & & & $\mathrm{C}_{3} \mathrm{H}_{7} \mathrm{O} \cdot \mathrm{CO}_{2}(103)$ & \\
\hline Propyl butyrate & 130 & $\mathrm{C}_{3} \mathrm{H}_{7} \mathrm{COO}^{-}(87)$ & 0.30 & & & $\mathrm{C}_{3} \mathrm{H}_{7} \mathrm{COO}^{-}(87)$ & 0.80 \\
\hline \multirow{3}{*}{$\mathrm{C}_{3} \mathrm{H}_{7} \mathrm{COOC}_{3} \mathrm{H}_{7}$} & 99.0 & M-1 (129) & 0.70 & & & M-1 (129) & 0.08 \\
\hline & $105-66-8$ & $M-1 . \mathrm{CO}_{2}(173)$ & & & & $\mathrm{C}_{3} \mathrm{H}_{7} \mathrm{O}^{-}(59)$ & 0.12 \\
\hline & & & & & & $\mathrm{C}_{3} \mathrm{H}_{7} \mathrm{O} \cdot \mathrm{CO}_{2}(103)$ & \\
\hline \multirow{5}{*}{$\begin{array}{c}\text { Butyl formate } \\
\mathrm{HCOOC}_{4} \mathrm{H}_{9}\end{array}$} & 102 & $\mathrm{HCOO}^{-}(45)$ & 0.87 & $\mathrm{HCOO}^{-}(45)$ & 1.0 & $\mathrm{HCOO}^{-}(45)$ & 0.81 \\
\hline & $\geq 97.0$ & $\mathrm{C}_{4} \mathrm{H}_{9} \mathrm{O}^{-}(73)$ & 0.13 & & & $\mathrm{C}_{4} \mathrm{H}_{9} \mathrm{O}^{-}(73)$ & 0.19 \\
\hline & $592-84-7$ & $\mathrm{C}_{4} \mathrm{H}_{9} \mathrm{O} \cdot \mathrm{H}_{2} \mathrm{O}(91)$ & & & & $\mathrm{C}_{4} \mathrm{H}_{9} \mathrm{O}: \mathrm{H}_{2} \mathrm{O}(91)$ & \\
\hline & & $\mathrm{C}_{4} \mathrm{H}_{9} \mathrm{O}: \mathrm{CO}_{2}(117)$ & & & & $\mathrm{C}_{4} \mathrm{H}_{9} \mathrm{O}: \mathrm{CO}_{2}(117)$ & \\
\hline & & $\mathrm{C}_{4} \mathrm{H}_{9} \mathrm{O}: \mathrm{C}_{4} \mathrm{H}_{9} \mathrm{OH}(147)$ & & & & $\mathrm{C}_{4} \mathrm{H}_{9} \mathrm{O}: \mathrm{C}_{4} \mathrm{H}_{9} \mathrm{OH}(147)$ & \\
\hline \multirow{4}{*}{$\mathrm{CH}_{3} \mathrm{COOC}_{4} \mathrm{H}_{9}$} & 116 & $\mathrm{CH}_{3} \mathrm{COO}^{-}(59)$ & 0.19 & & & $\mathrm{CH}_{3} \mathrm{COO}^{-}(59)$ & 0.73 \\
\hline & $\geq 99.7$ & M-1 (115) & 0.81 & & & M-1 (115) & 0.17 \\
\hline & $123-86-4$ & & & & & $\mathrm{C}_{4} \mathrm{H}_{9} \mathrm{O}^{-}(73)$ & 0.10 \\
\hline & & & & & & $\mathrm{C}_{4} \mathrm{H}_{9} \mathrm{O} \cdot \mathrm{CO}_{2}(117)$ & \\
\hline Butyl propionate & 130 & $\mathrm{C}_{2} \mathrm{H}_{5} \mathrm{COO}^{-}(73)$ & 0.45 & & & $\mathrm{C}_{2} \mathrm{H}_{5} \mathrm{COO}^{-}(73)$ & nd \\
\hline \multirow[t]{3}{*}{$\mathrm{C}_{2} \mathrm{H}_{5} \mathrm{COOC}_{4} \mathrm{H}_{9}$} & $\geq 97.0$ & M-1 (129) & 0.55 & & & M-1 (129) & 0.08 \\
\hline & $590-01-2$ & $M-1 . \mathrm{CO}_{2}(173)$ & & & & $\mathrm{C}_{4} \mathrm{H}_{9} \mathrm{O}^{-}(73)$ & nd \\
\hline & & & & & & $\mathrm{C}_{4} \mathrm{H}_{9} \mathrm{O} \cdot \mathrm{CO}_{2}(117)$ & \\
\hline Butyl butyrate & 144 & $\mathrm{C}_{3} \mathrm{H}_{7} \mathrm{COO}^{-}(87)$ & 0.22 & & & $\mathrm{C}_{3} \mathrm{H}_{7} \mathrm{COO}^{-}(87)$ & 0.80 \\
\hline \multirow{3}{*}{$\mathrm{C}_{3} \mathrm{H}_{7} \mathrm{COOC}_{4} \mathrm{H}_{9}$} & $\geq 99.7$ & M-1 (143) & 0.78 & & & M-1 (143) & 0.07 \\
\hline & $109-21-7$ & & & & & $\mathrm{C}_{4} \mathrm{H}_{9} \mathrm{O}^{-}(73)$ & 0.13 \\
\hline & & & & & & $\mathrm{C}_{4} \mathrm{H}_{9} \mathrm{O} \cdot \mathrm{CO}_{2}(117)$ & \\
\hline
\end{tabular}

In italic, secondary product ions. BR: branching ratio. 
1.4 - Nitrite $\left(\mathrm{NO}_{2}{ }^{-}\right)$and nitrate ions $\left(\mathrm{NO}_{3}{ }^{-}\right)$reactions

Carboxylic acids react with $\mathrm{NO}_{2}^{-}$precursor ion resulting in two product ions from two different reaction pathways. In addition to the deprotonation (reaction (Eq 19)), an ion-molecule association reaction is observed (reaction $(\mathrm{Eq} 20)$ ). This last one is the major reaction with branching ratio from 64 to $99 \%$ (Table 1).

$$
\begin{aligned}
& \mathrm{NO}_{2}{ }^{-}+\mathrm{RCOOH} \rightarrow \mathrm{RCOO}^{-}+\mathrm{HNO}_{2} \\
& \mathrm{NO}_{2}{ }^{-}+\mathrm{RCOOH}+\mathrm{N}_{2} \rightarrow \mathrm{RCOOH} \cdot \mathrm{NO}_{2}{ }^{-}+\mathrm{N}_{2}
\end{aligned}
$$

The calculated energies of the reactions 19 and 20, from the starting carboxylic acids to the final $\mathrm{RCOO}^{-}$anions or $\mathrm{RCOOH} . \mathrm{NO}_{2}{ }^{-}$complexes, are summarized in Figure 1. In all cases, the formation of the RCOOH. $\mathrm{NO}_{2}{ }^{-}$complex is exergonic from -18.3 to $-11.9 \mathrm{kcal} \mathrm{mol}^{-1}$, while the deprotonation is endergonic (from 4.31 to $8.21 \mathrm{kcal} \mathrm{mol}^{-1}$ ). Thus, the complex formation is thermodynamically more favored explaining that $\mathrm{RCOOH} . \mathrm{NO}_{2}{ }^{-}$product ions are largely in the majority of the reaction of the carboxylic acids with the $\mathrm{NO}_{2}{ }^{-}$precursor ion with high branching ratios. The $\mathrm{RCOO}^{-}$product ions are still experimentally observed due to the experimental conditions in the flow tube $(393 \mathrm{~K})$ providing enough energy to permit the reaction. The distribution between the two reaction pathways (reactions 19 and 20) depends on the activation energies, thermodynamic process, and energy provided by the experimental conditions.

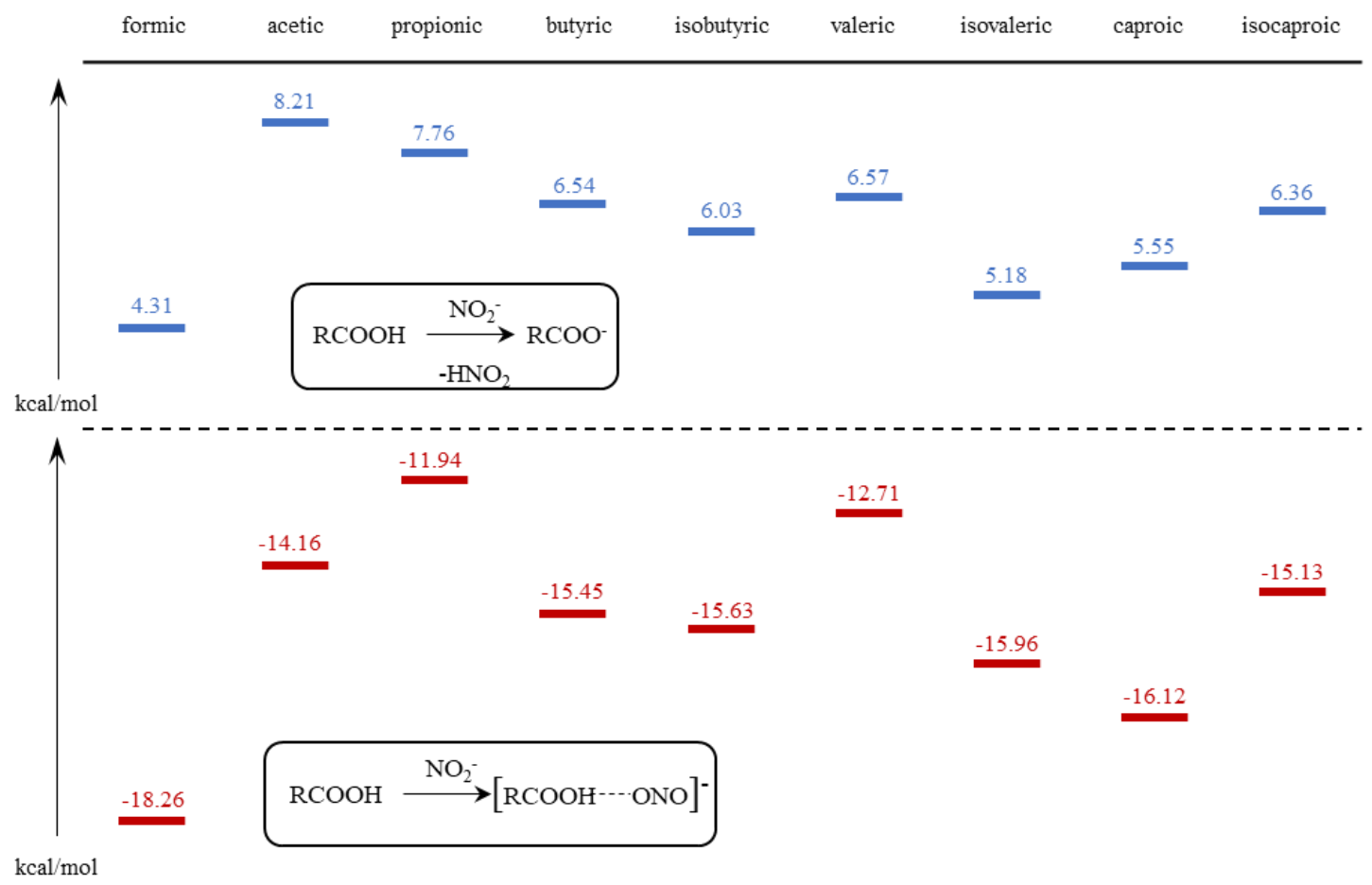

Figure 1: Calculated Gibbs energies ( $k \mathrm{cal} / \mathrm{mol}$ ) for the reactions of $\mathrm{NO}_{2}{ }^{-}$reagent ion with carboxylic acids leading to $\mathrm{RCOO}^{-}$ anion or RCOOH. $\mathrm{NO}_{2}{ }^{-}$complex. Energies are calculated in the gas phase, using MP2 methods coupled with the 6$311++g(d, p)$. The reagents $\left(M\right.$ and $\left.\mathrm{NO}_{2}^{-}\right)$are considered as origin. 
No reaction was experimentally observed between $\mathrm{NO}_{3}{ }^{-}$precursor ion and carboxylic acids and between esters and $\mathrm{NO}_{2}^{-}$and $\mathrm{NO}_{3}^{-}$precursor ions. If such reactions appear theoretically possible, they are probably too slow to allow the detection of product ions. Note that collisional constant rates are lower with the $\mathrm{NO}_{2}{ }^{-}$and $\mathrm{NO}_{3}{ }^{-}$ions than with the other negative precursor ions (Table 2).

We can also note that the formation of the complex with acids is less thermodynamically favorable than that involving $\mathrm{NO}_{2}^{-}$(e.g. $-9.10 \mathrm{vs}-14.16 \mathrm{kcal} \mathrm{mol}^{-1}$ for acetic acid and $-9.36 \mathrm{vs}$ $-15.96 \mathrm{kcal} \mathrm{mol}^{-1}$ for isovaleric acid).

\section{2 - Reaction kinetics}

2.1 - Experimental rate constants $k$ and calculated collisional rate constants $k_{c}$

One of the major strengths of the SIFT-MS technology is the possibility of quantification without calibration. Indeed, quantification by SIFT-MS is based on the knowledge of the reactivity constant $k$ (see Eq 1). While in positive ionisation a large database exists, there is currently only a few scattered data in negative ionisation. The determination of this constant is therefore a key element for quantitative analyses, as important as the knowledge of the ions produced from the reaction in the flow tube. In this work, we have therefore determined these reactivity constants experimentally for the carboxylic acids and esters studied with the various negative precursor ions from calibration ranges (see experimental section). The reactivity constants thus determined are shown in Table 2.

Table 2. Experimental rate constants $k$ (in $N_{2}$ ) and calculated collisional rate constants $k_{c}$ for the reactions of carboxylic acids and negative precursor ions in SIFT-MS.

\begin{tabular}{|c|c|c|c|c|c|c|c|c|}
\hline \multirow{2}{*}{ Compound } & \multirow{2}{*}{ MW } & \multirow{2}{*}{$\alpha^{\mathrm{a}}$} & \multirow{2}{*}{$\mu_{\mathrm{r}}^{\mathrm{a}}$} & $\mathrm{OH}^{-}$ & $0^{\bullet-}$ & $\mathrm{O}_{2}{ }^{\bullet-}$ & $\mathrm{NO}_{2}^{-}$ & $\mathrm{NO}_{3}^{-}$ \\
\hline & & & & $k\left[k_{c}\right]^{\mathbf{b}}$ & $k\left[k_{c}\right]^{\mathbf{b}}$ & $k\left[k_{c}\right]^{\mathbf{b}}$ & $k\left[k_{c}\right]^{\mathbf{b}}$ & {$\left[k_{c}\right]^{\mathbf{b}}$} \\
\hline Formic acid & 46 & 2.98 & 1.67 & $0.8[2.56]^{\mathrm{c}}$ & $0.5[2.62]^{c}$ & $2.3[2.07]^{\mathrm{c}}$ & $0.2[1.88]^{\mathrm{c}}$ & $\mathrm{ND}$ [1.75] \\
\hline Acetic acid & 60 & 4.65 & 1.97 & $1.2[2.96]^{\mathrm{c}}$ & $0.2[3.04]^{\mathrm{c}}$ & $1.3[2.36]^{\mathrm{c}}$ & $0.2[2.12]^{\mathrm{c}}$ & ND [1.96] \\
\hline Propionic acid & 74 & 6.33 & 1.83 & $1.3[2.89]$ & $0.2[2.97]$ & $1.6[2.28]$ & $0.2[2.02]$ & $\mathrm{ND}$ [1.85] \\
\hline Butyric acid & 88 & 8.14 & 2.17 & $1.0[3.33]^{\mathrm{c}}$ & $0.3[3.42]^{\mathrm{c}}$ & $1.7[2.60]^{\mathrm{c}}$ & $0.5[2.29]^{\mathrm{c}}$ & ND [2.08] \\
\hline Isobutyric acid & 88 & 8.08 & 1.92 & $1.1[3.07]$ & $0.2[3.15]$ & 1.6 [2.39] & $0.4[2.11]$ & $\mathrm{ND}$ [1.92] \\
\hline Valeric acid & 102 & 11.54 & 1.78 & $1.0[3.11]$ & $0.2[3.19]$ & $1.4[2.40]$ & $0.5[2.11]$ & ND [1.91] \\
\hline Isovaleric acid & 102 & 9.86 & 2.20 & $1.3[3.43]$ & $0.3[3.52]$ & $2.1[2.65]$ & $0.6[2.33]$ & $\mathrm{ND}$ [2.11] \\
\hline Caproic acid & 116 & 11.63 & 2.16 & $1.3[3.46]^{\mathrm{c}}$ & $0.2[3.55]^{\mathrm{c}}$ & $1.2[2.66]^{\mathrm{c}}$ & $1.0[2.32]^{\mathrm{c}}$ & $\mathrm{ND}$ [2.10] \\
\hline Isocaproic acid & 116 & 11.57 & 1.83 & $0.5[3.13]$ & $0.1[3.22]$ & $0.8[2.41]$ & $0.6[2.10]$ & ND [1.90] \\
\hline Methyl formate & 60 & $\mathrm{NC}$ & $\mathrm{NC}$ & $1.3[\mathrm{NC}]$ & $0.1[\mathrm{NC}]$ & ND & ND & ND \\
\hline Methyl acetate & 74 & $\mathrm{NC}$ & $\mathrm{NC}$ & $1.5[\mathrm{NC}]$ & $0.1[\mathrm{NC}]$ & ND & ND & ND \\
\hline Methyl propionate & 88 & 8.05 & 2.08 & $1.7[3.24]$ & $0.1[3.32]$ & $\mathrm{ND}$ [2.52] & $\mathrm{ND}[2.22]$ & $\mathrm{ND}[2.03]$ \\
\hline Methyl butyrate & 102 & $\mathrm{NC}$ & $\mathrm{NC}$ & $2.2[\mathrm{NC}]$ & $0.1[\mathrm{NC}]$ & ND & ND & ND \\
\hline Methyl valerate & 116 & 11.63 & 2.11 & $2.2[3.41]$ & $0.1[3.50]$ & {$[2.62]$} & [2.29] & [2.07] \\
\hline
\end{tabular}




\begin{tabular}{|c|c|c|c|c|c|c|c|c|}
\hline Methyl caproate & 130 & 13.43 & 2.11 & $1.6[3.49]$ & 0.1 [3.59] & [2.67] & [2.32] & [2.09] \\
\hline Ethyl formate & 74 & $\mathrm{NC}$ & $\mathrm{NC}$ & $2.1[\mathrm{NC}]$ & $0.1[\mathrm{NC}]$ & ND & ND & ND \\
\hline Ethyl acetate & 88 & $\mathrm{NC}$ & $\mathrm{NC}$ & $2.0[\mathrm{NC}]$ & $0.1[\mathrm{NC}]$ & ND & ND & ND \\
\hline Ethyl propionate & 102 & 9.83 & 2.00 & $\mathrm{NM}$ [3.23] & $\mathrm{NM}$ [3.31] & $\mathrm{NM}[2.50]$ & $\mathrm{NM}$ [2.19] & NM [1.98] \\
\hline Ethyl butyrate & 116 & 11.57 & 1.88 & $2.0[3.18]$ & $0.1[3.27]$ & $\mathrm{ND}[2.45]$ & $\mathrm{ND}$ [2.13] & $\mathrm{ND}$ [1.93] \\
\hline Ethyl valerate & 130 & 13.35 & 1.90 & $1.5[3.27]$ & $0.1[3.36]$ & $\mathrm{ND}[2.50]$ & ND [2.17] & ND [1.96] \\
\hline Ethyl isovalerate & 130 & $\mathrm{NC}$ & $\mathrm{NC}$ & $1.5[\mathrm{NC}]$ & $0.1[\mathrm{NC}]$ & ND & ND & ND \\
\hline Ethyl caproate & 144 & 15.07 & 1.82 & $1.3[3.26]$ & $0.1[3.35]$ & $\mathrm{ND}[2.48]$ & $\mathrm{ND}$ [2.15] & $\mathrm{ND}$ [1.93] \\
\hline Propyl formate & 88 & $\mathrm{NC}$ & $\mathrm{NC}$ & $1.5[\mathrm{NC}]$ & $0.3[\mathrm{NC}]$ & ND & ND & ND \\
\hline Propyl acetate & 102 & $\mathrm{NC}$ & $\mathrm{NC}$ & $1.5[\mathrm{NC}]$ & $0.3[\mathrm{NC}]$ & ND & ND & ND \\
\hline Propyl propionate & 116 & 11.55 & 2.07 & $2.8[3.37]$ & $0.3[3.46]$ & ND [2.59] & $\mathrm{ND}[2.26]$ & $\mathrm{ND}$ [2.04] \\
\hline Propyl butyrate & 130 & 13.33 & 1.96 & 2.5 [3.33] & $0.2[3.42]$ & $\mathrm{ND}[2.55]$ & $\mathrm{ND}$ [2.22] & ND [1.99] \\
\hline Propyl valerate & 144 & 15.13 & 2.01 & NM [3.45] & NM [3.54] & NM [2.63] & $\mathrm{NM}$ [2.28] & NM [2.04] \\
\hline Propyl caproate & 158 & 16.93 & 1.92 & NM [3.43] & $\mathrm{NM}$ [3.52] & NM [2.60] & $\mathrm{NM}$ [2.25] & $\mathrm{NM}$ [2.01] \\
\hline Butyl formate & 102 & $\mathrm{NC}$ & $\mathrm{NC}$ & $1.8[\mathrm{NC}]$ & $0.3[\mathrm{NC}]$ & ND & ND & ND \\
\hline Butyl acetate & 116 & $\mathrm{NC}$ & $\mathrm{NC}$ & $1.5[\mathrm{NC}]$ & $0.1[\mathrm{NC}]$ & ND & ND & ND \\
\hline Butyl propionate & 130 & 13.35 & 2.08 & $1.5[3.45]$ & $0.1[3.54]$ & ND [2.64] & $\mathrm{ND}$ [2.29] & $\mathrm{ND}$ [2.06] \\
\hline Butyl butyrate & 144 & 15.03 & 2.09 & $1.0[3.53]$ & $0.1[3.62]$ & ND [2.69] & $\mathrm{ND}$ [2.33] & ND [2.09] \\
\hline Butyl valerate & 158 & 16.79 & 2.17 & NM [3.67] & NM [3.77] & NM [2.79] & NM [2.41] & NM [2.16] \\
\hline Butyl caproate & 172 & 18.44 & 2.20 & NM [3.76] & NM [3.86] & NM [2.85] & NM [2.45] & NM [2.19] \\
\hline
\end{tabular}

The collisional rate constant $k_{c}$ is calculated according to parametrized trajectory calculations using the dipole moment $\mu_{r}$ and the polarizability $\alpha$ of the neutral, and the reduced mass of the collisional ion-molecule partners. Thus, the calculated $k_{c}$ are higher with $\mathrm{O}^{\bullet-}$ precursor ion for which the ion-molecule complex involved have the lowest reduced masses. The collisional rate constant $k_{c}$ also increases with the polarizability $\alpha$, i.e. generally with the molecular weight of the compounds.

In gas phase, reactions occur under certain particular conditions: first, molecules must collide to react, but every collision is not successful; indeed, the molecules also need to collide with the "right" orientation and with "enough" energy. Thus, the collisional rate constant $k_{c}$ reflects the collision possibilities of the partners, while the reaction rate constant $k$ reflects the effective collision i.e. collision leading to reaction. For carboxylic acids as for esters, experimental reaction rate constants $k$ are much lower than collisional rate constants $k_{c}$ (Table 2). It means that not all collisions in the SIFT-MS flow tube are effective.

In addition to the analyte reaction with the reagent, other reactions can occur simultaneously in the flow tube. These side reactions are notably linked to the presence of water and other interfering components in the flow tube. In a previous work, we listed the product ions from the reaction of the negative precursor ions with the permanent components of an air matrix 
(oxygen, water and carbon dioxide) [32]. These interferent ions are not without consequences on the kinetics of the studied ion-molecule reactions. For instance, the hydrated hydroxide ions $\mathrm{OH}^{-} . \mathrm{H}_{2} \mathrm{O}$ and $\mathrm{OH}^{-} .\left(\mathrm{H}_{2} \mathrm{O}\right)_{2}$ from the reaction of water with $\mathrm{OH}^{-}$precursor also act as reagent ions and thus react with carboxylic acids and esters (reactions 9 and 10). In this particular case, the reaction rate constant $k$ experimentally determined corresponds to an "apparent reaction rate" considering all the reactions leading to the formation of the product ions and not only the reaction of carboxylic acids / esters with $\mathrm{OH}^{-}$. Based on experimental observations and collision constants, the reactions of the hydrated hydroxide ions with carboxylic acids / esters are slower than those with hydroxide ion, so the overall reaction is slowed down by these reactions (Table S1).

We also previously noticed that the reactions can lead to the formation of radicals or to $\mathrm{OH}^{-}$ion products. These products are "invisible" in SIFT-MS and the reactions leading to their formation are neglected in the determination of the experimental reaction rate constants $k$.

It is also obvious that the flow tube parameters have a significant influence on the measured rate constant $k$, in particular the temperature. In fact, the flow tube temperature has several effects on the reaction rate. On the one hand, it influences the speed of molecules in the flow tube and therefore the number of collisions. On the other hand, the proportion of molecules having an energy higher than the activation energy increases with the temperature.

Table S1. Calculated collisional rate constants $k_{c}$ for the reactions of carboxylic acids and esters with hydroxide ion and hydrated hydroxide ions $\left(10^{-9} \mathrm{~cm}^{3} \mathrm{~s}^{-1}\right)$

\begin{tabular}{|c|c|c|c|}
\hline Compound & $\mathbf{O H}^{-}$ & $\mathrm{OH}^{-} \cdot \mathrm{H}_{2} \mathrm{O}$ & $\mathrm{OH}^{-} \cdot\left(\mathrm{H}_{2} \mathrm{O}\right)_{2}$ \\
\hline Formic acid & 2.56 & 2.02 & 1.82 \\
\hline Acetic acid & 2.96 & 2.30 & 2.04 \\
\hline Propionic acid & 2.89 & 2.21 & 1.94 \\
\hline Butyric acid & 3.33 & 2.51 & 2.19 \\
\hline Isobutyric acid & 3.07 & 2.32 & 2.01 \\
\hline Valeric acid & 3.11 & 2.32 & 2.01 \\
\hline Isovaleric acid & 3.43 & 2.57 & 2.22 \\
\hline Caproic acid & 3.46 & 2.57 & 2.21 \\
\hline Isocaproic acid & 3.13 & 2.33 & 2.00 \\
\hline Methyl propionate & 3.24 & 2.44 & 2.08 \\
\hline Methyl valerate & 3.41 & 2.53 & 2.13 \\
\hline Methyl caproate & 3.49 & 2.58 & 2.15 \\
\hline Ethyl propionate & 3.23 & 2.41 & 2.04 \\
\hline Ethyl butyrate & 3.18 & 2.36 & 1.98 \\
\hline Ethyl valerate & 3.27 & 2.41 & 2.01 \\
\hline Ethyl caproate & 3.26 & 2.40 & 1.99 \\
\hline Propyl propionate & 3.37 & 2.50 & 2.10 \\
\hline Propyl butyrate & 3.33 & 2.46 & 2.05 \\
\hline
\end{tabular}




\begin{tabular}{cccc} 
Propyl valerate & 3.45 & 2.53 & 2.10 \\
Propyl caproate & 3.43 & 2.51 & 2.07 \\
\hline Butyl propionate & 3.45 & 2.55 & 2.12 \\
Butyl butyrate & 3.53 & 2.59 & 2.15 \\
Butyl valerate & 3.67 & 2.68 & 2.22 \\
Butyl caproate & 3.76 & 2.74 & 2.26 \\
\hline
\end{tabular}

\section{2 - Quantitative analysis}

The knowledge of the rate constants $k$ is a key parameter in SIFT-MS quantification. The rate constant $k$ must be high enough $\left(>10^{-10} \mathrm{~cm}^{3} \mathrm{~s}^{-1}\right)$ for an accurate quantification. This condition is fulfilled for studied carboxylic acids and esters.

The rate constants of carboxylic acids are fast enough to allow a good quantification with $\mathrm{OH}^{-}$ and $\mathrm{O}_{2}{ }^{\bullet-}$ precursor ions with values from 0.5 to 1.3 and from 0.8 to $2.310^{-9} \mathrm{~cm}^{3} \mathrm{~s}^{-1}$, respectively. When comparing the negative to the positive mode, the reactions rate constants of carboxylic acids are slightly lower but of the same order of magnitude (from 2.2 to $3.010^{-9} \mathrm{~cm}^{3} \mathrm{~s}^{-1}$ with $\mathrm{H}_{3} \mathrm{O}^{+}$precursor ion, see Table $\mathrm{S} 2$, supporting information). $\mathrm{O}^{\bullet-}$ and $\mathrm{NO}_{2}^{-}$precursor ions can also be used for the quantification of carboxylic acids but the reactivity is much lower, with rate constants in the range of $10^{-10} \mathrm{~cm}^{3} \mathrm{~s}^{-1}$, which will mean lower sensitivity (see LOD, Table 3). Thus, $\mathrm{OH}^{-}$and $\mathrm{O}_{2}{ }^{\bullet-}$ precursor ions must be favored for a sensitive detection and accurate quantification of carboxylic acids in negative ionization. Limits of detection of carboxylic acids with $\mathrm{OH}^{-}$and $\mathrm{O}_{2}{ }^{\bullet-}$ are of the same order of magnitude as with $\mathrm{NO}^{+}$and $\mathrm{O}_{2}{ }^{+}$precursor ions and lower than with $\mathrm{H}_{3} \mathrm{O}^{+}$precursor ion (Table 3).

For the quantification of esters, only $\mathrm{OH}^{-}$and $\mathrm{O}^{\bullet-}$ precursor ions have reaction rates high enough. The sensitivity is higher with $\mathrm{OH}^{-}$precursor ions with reaction rate constants from 1.0 to $2.810^{-9} \mathrm{~cm}^{3} \mathrm{~s}^{-1}$, versus 0.1 to $0.310^{-9} \mathrm{~cm}^{3} \mathrm{~s}^{-1}$ with $\mathrm{O}^{\bullet-}$. This translates experimentally into much lower detection limits with $\mathrm{OH}^{-}$(from 0.4 to $6.2 \mathrm{ppb}$ ) than with $\mathrm{O}^{\bullet-}$ ion (from 0.6 to 30.6 $\mathrm{ppb}$ ). The reaction rate of esters with the $\mathrm{OH}^{-}$hydroxide ion is comparable to those in positive ionization (see Table S2, supporting information). Some product ions were experimentally detected in reaction of esters with $\mathrm{O}_{2}{ }^{-}$(reaction 13, section 1.2) but the reaction rate seems too low to be measured. Limits of detection of individual pure compounds in nitrogen were experimentally determined (Table 3). However, we draw the reader's attention to the fact that this detection limit can be modulated by the instrument settings (pressure and temperature of the flow tube but also the ion source efficiency).

Table 3. Experimental LOD in SIM mode SIFT-MS (in ppbV).

\begin{tabular}{ccccccccc}
\hline Compounds & $\mathbf{H}_{3} \mathbf{O}^{+}$ & $\mathbf{N O}^{+}$ & $\mathbf{O}_{2}{ }^{+}$ & $\mathbf{O H}^{-}$ & $\mathbf{O}_{2}^{-}$ & $\mathbf{O}^{-}$ & $\mathbf{N O}_{2}^{-}$ & $\mathbf{N O}^{-}$ \\
\hline Formic acid & & Carboxylic acids & & & & \\
Acetic acid & 67.7 & & 13.2 & 15.5 & 7.5 & 11.7 & \\
Propionic acid & 9.4 & 2.0 & 6.7 & 5.6 & 3.3 & 7.7 & 5.8 & \\
Butyric acid & 3.8 & 1.0 & 0.5 & 1.5 & 1.3 & 4.7 & 4.2 & \\
Isobutyric acid & 3.8 & 0.4 & 1.7 & 0.9 & 0.6 & 1.2 & 4.2 &
\end{tabular}




\begin{tabular}{|c|c|c|c|c|c|c|c|}
\hline Valeric acid & 12.6 & 0.4 & 4.3 & 2.9 & 1.9 & 7.6 & 2.5 \\
\hline Isovaleric acid & 11.3 & 0.7 & & 2.3 & 2.7 & 6.6 & 1.7 \\
\hline Caproic acid & 4.1 & 0.2 & & 0.5 & 1.1 & 1.6 & 1.9 \\
\hline Isocaproic acid & & & & 1.3 & 1.5 & 3.7 & 3.0 \\
\hline \multicolumn{8}{|c|}{ Esters } \\
\hline Methyl formate & 9.3 & 2.9 & 6.3 & 2.2 & & 12.5 & \\
\hline Methyl acetate & 4.2 & 2.9 & 2.6 & 0.8 & & 13.9 & \\
\hline Methyl propionate & 3.5 & 2.6 & 2.7 & 0.7 & & 11.1 & \\
\hline Methyl butyrate & 10.9 & 1.9 & 0.8 & 0.4 & & 15.3 & \\
\hline Methyl valerate & 3.4 & 0.8 & 1.2 & 0.4 & & 10.9 & \\
\hline Methyl caproate & 0.7 & 2.7 & 1.0 & 1.0 & & 10.7 & \\
\hline Ethyl formate & 2.1 & 0.3 & 3.2 & 3.3 & & 30.6 & \\
\hline Ethyl acetate & 3.3 & 0.5 & 7.2 & 0.6 & & 0.6 & \\
\hline Ethyl butyrate & 4.2 & 0.9 & 0.6 & 0.5 & & 8.7 & \\
\hline Ethyl valerate & 2.7 & 0.8 & 0.7 & 1.2 & & 7.3 & \\
\hline Ethyl 3-methylbutanoate & 2.2 & 0.7 & 0.8 & 1.3 & & 8.6 & \\
\hline Ethyl caproate & 5.4 & 0.5 & & 1.0 & & 9.3 & \\
\hline Propyl formate & & & & 6.2 & & 11.3 & \\
\hline Propyl acetate & 9.2 & 1.0 & 0.4 & 0.6 & & 0.6 & \\
\hline Propyl propionate & 2.9 & 0.3 & 0.1 & 0.4 & & 2.8 & \\
\hline Propyl butyrate & 2.3 & 1.3 & 1.3 & 0.7 & & 3.8 & \\
\hline Butyl formate & 1.9 & 6.1 & 3.7 & 5.1 & & 6.9 & \\
\hline Butyl acetate & 8.3 & 0.7 & 27.5 & 0.7 & & 0.7 & \\
\hline Butyl propionate & 1.4 & 1.8 & 0.2 & 1.6 & & 9.3 & \\
\hline Butyl butyrate & 1.2 & 1.6 & 1.8 & 1.1 & & 9.3 & \\
\hline
\end{tabular}

Table S2. Reaction rate constants $k$ and collisional rate constants $k_{c}$ for the reactions of carboxylic acids and positive precursor ions in SIFT-MS. Unit: $10^{-9} \mathrm{~cm}^{3} \mathrm{~s}^{-1}$.

\begin{tabular}{|c|c|c|c|c|c|c|c|c|}
\hline \multirow{2}{*}{ Compound } & \multirow{2}{*}{ MW } & \multicolumn{2}{|c|}{$\mathrm{H}_{3} \mathrm{O}^{+}$} & \multicolumn{2}{|c|}{$\mathrm{NO}^{+}$} & \multicolumn{2}{|c|}{$\mathrm{O}_{2}^{+}$} & \multirow{2}{*}{ Reference } \\
\hline & & $k$ & $k_{c}$ & $k$ & $k_{c}$ & $k$ & $k_{c}$ & \\
\hline Formic acid & 46 & 2.2 & 2.2 & $<0.1$ & 1.9 & 2.0 & 1.9 & [37] \\
\hline Acetic acid & 60 & 2.6 & 2.6 & 0.9 & 2.2 & 2.3 & 2.2 & [37] \\
\hline Propionic acid & 74 & 2.7 & 2.7 & 1.5 & 2.3 & 2.2 & 2.2 & [37] \\
\hline Butyric acid & 88 & 2.9 & 2.9 & 1.9 & 2.4 & 2.1 & 2.4 & [37] \\
\hline Isobutyric acid & 88 & 2.9 & 2.9 & 2.5 & 2.4 & 2.5 & 2.4 & [37] \\
\hline Valeric acid & 102 & 2.9 & 2.9 & 2.4 & 2.4 & 2.4 & 2.4 & [37] \\
\hline Isovaleric acid & 102 & 3.0 & 3.0 & 2.5 & & & & LabSyft sofware \\
\hline Caproic acid & 116 & 3.0 & 3.0 & 2.5 & & & & LabSyft sofware \\
\hline Isocaproic acid & 116 & & & & & & & \\
\hline Methyl formate & 60 & 2.7 & 2.7 & 0.5 & 2.3 & 2.2 & 2.3 & [37] \\
\hline Methyl acetate & 74 & 2.8 & 2.8 & 1.6 & 2.3 & 2.4 & 2.3 & [37] \\
\hline Methyl propionate & 88 & 2.9 & 2.9 & 2.1 & 2.4 & 2.0 & 2.4 & [37] \\
\hline Methyl butyrate & 102 & 2.9 & 2.9 & 2.4 & 2.4 & 2.4 & 2.4 & [37] \\
\hline Methyl valerate & 116 & 3.0 & & 1.5 & & 1.6 & & LabSyft sofware \\
\hline Methyl caproate & 130 & 3.0 & & 2.1 & & 1.7 & & LabSyft sofware \\
\hline Ethyl formate & 74 & 3.0 & 3.0 & 1.6 & 2.5 & 2.5 & 2.5 & [37] \\
\hline Ethyl acetate & 88 & 2.9 & 2.9 & 2.1 & 2.4 & 2.4 & 2.4 & {$[37]$} \\
\hline Ethyl propionate & 102 & 2.9 & 2.9 & 2.5 & 2.4 & 2.5 & 2.4 & [37] \\
\hline Ethyl butyrate & 116 & 3.0 & 3.2 & 2.4 & 2.7 & 2.5 & 2.6 & {$[38]$} \\
\hline
\end{tabular}




\begin{tabular}{|c|c|c|c|c|c|c|c|c|}
\hline Ethyl valerate & 130 & 3.0 & 3.0 & 2.5 & \multicolumn{3}{|c|}{2.5} & \multirow[t]{2}{*}{ LabSyft sofware } \\
\hline Ethyl isovalerate & 130 & & & & & & & \\
\hline Ethyl caproate & 144 & 3.0 & 3.0 & 2.5 & & 4.8 & & LabSyft sofware \\
\hline Propyl formate & 88 & & & & & & & \\
\hline Propyl propionate & 116 & 3.3 & 3.0 & 2.2 & 2.5 & 2.2 & 2.5 & {$[38]$} \\
\hline Propyl butyrate & 130 & 3.2 & 3.2 & 2.0 & 2.6 & 1.8 & 2.6 & {$[38]$} \\
\hline Propyl valerate & 144 & & & & & & & \\
\hline Propyl caproate & 158 & & & & & & & \\
\hline Butyl formate & 102 & 3.0 & 3.5 & 1.3 & 2.9 & 2.2 & 2.8 & {$[38]$} \\
\hline Butyl acetate & 116 & 2.9 & 3.3 & 2.0 & 2.8 & 2.3 & 2.7 & {$[38]$} \\
\hline Butyl propionate & 130 & 2.9 & 3.1 & 1.8 & 2.6 & 2.4 & 2.5 & {$[38]$} \\
\hline Butyl butyrate & 144 & 3.1 & 3.2 & 2.2 & 2.7 & 2.9 & 2.6 & {$[38]$} \\
\hline Butyl valerate & 158 & & & & & & & \\
\hline Butyl caproate & 172 & & & & & & & \\
\hline
\end{tabular}

\section{Conclusions}

Direct Injection Mass Spectrometry instruments are currently becoming more relevant for providing rapid measurements of a wide variety of volatile organic compounds in several applications fields: environment, agro-food product quality or health. From the first PTR-MS instruments capable of quantifying analytes thanks to $\mathrm{H}_{3} \mathrm{O}^{+}$precursor ion, many instrumental developments increase the selectivity of these instruments by adding other reactive cations $\left(\mathrm{NO}^{+}\right.$and $\left.\mathrm{O}_{2}{ }^{+}\right)$. More recently, new plasma sources in SIFT-MS allow an extension of the precursor ions to anions, opening new reactions ways. However, the lack of data on reactivities and rate constants with these new reactive anions is a limitation to the deployment of these new instruments.

The present study enhances the knowledge and the database on the reactivity of carboxylic acids and esters with negative precursor ions $\left(\mathrm{OH}^{-}, \mathrm{O}^{\bullet-}, \mathrm{O}_{2}{ }^{\bullet-}, \mathrm{NO}_{2}^{-}\right.$and $\left.\mathrm{NO}_{3}{ }^{-}\right)$. Product ions, reaction pathways and reaction rate constants needed for the quantification of carboxylic acids and esters with the negative ionization mode are now available. This preliminary work opens a new way for the quantification of short chain fatty acids that are sometimes difficult to quantify in complex matrices with traditional separative methods.

Carboxylic acids, alcohol and esters are organic molecules involved in several metabolic pathways and are usually found in many mixtures. However, this work illustrates also a limitation of DIMS technics because of interferences between product ions with same $\mathrm{m} / \mathrm{z}$ from different analytes (Table 1). However, we believe that the knowledge gained from identification of the product ions from the carboxylic acids and esters, and of other compounds, builds the database and makes it possible to be aware of these interference issues in SIFT-MS measurements. Besides, the detection of a conflict ion like -87 with $\mathrm{O}^{\bullet-}$ known to correspond to butyric acid and also to alkyl butyrate esters gives information on global metabolic pathways and may allow the quantification of the "global butyric derivatives".

\section{Acknowledgements}

Authors thank Conseil Régional Nouvelle Aquitaine (CRNA) for financial support of this work in the frame of POLLIMPACT project. 


\section{References}

[1] D. Smith, P. Španěl, SIFT-MS and FA-MS methods for ambient gas phase analysis: developments and applications in the UK, The Analyst. 140 (2015) 2573-2591. https://doi.org/10.1039/C4AN02049A.

[2] W.-T. Tsai, An overview of health hazards of volatile organic compounds regulated as indoor air pollutants, Reviews on Environmental Health. 34 (2019) 81-89. https://doi.org/10.1515/reveh-2018-0046.

[3] F. Buljubasic, G. Buchbauer, The scent of human diseases: a review on specific volatile organic compounds as diagnostic biomarkers: The scent of human diseases, Flavour and Fragrance Journal. 30 (2015) 5-25. https://doi.org/10.1002/ffj.3219.

[4] F. Biasioli, F. Gasperi, C. Yeretzian, T.D. Märk, PTR-MS monitoring of VOCs and BVOCs in food science and technology, TrAC Trends in Analytical Chemistry. 30 (2011) 968-977. https://doi.org/10.1016/j.trac.2011.03.009.

[5] Y.Y. Broza, P. Mochalski, V. Ruzsanyi, A. Amann, H. Haick, Hybrid Volatolomics and Disease Detection, Angewandte Chemie International Edition. 54 (2015) 11036-11048. https://doi.org/10.1002/anie.201500153.

[6] S. Giannoukos, A. Agapiou, B. Brkić, S. Taylor, Volatolomics: A broad area of experimentation, Journal of Chromatography B. 1105 (2019) 136-147. https://doi.org/10.1016/j.jchromb.2018.12.015.

[7] B. Buszewski, M. Kęsy, T. Ligor, A. Amann, Human exhaled air analytics: biomarkers of diseases, Biomedical Chromatography. 21 (2007) 553-566. https://doi.org/10.1002/bmc.835.

[8] C. Turner, B. Parekh, C. Walton, P. Španěl, D. Smith, M. Evans, An exploratory comparative study of volatile compounds in exhaled breath and emitted by skin using selected ion flow tube mass spectrometry, Rapid Commun. Mass Spectrom. 22 (2008) 526-532. https://doi.org/10.1002/rcm.3402.

[9] X. Sun, K. Shao, T. Wang, Detection of volatile organic compounds (VOCs) from exhaled breath as noninvasive methods for cancer diagnosis, Analytical and Bioanalytical Chemistry. (2015). https://doi.org/10.1007/s00216-015-9200-6.

[10] L. Guo, C. Wang, C. Chi, X. Wang, S. Liu, W. Zhao, C. Ke, G. Xu, E. Li, Exhaled breath volatile biomarker analysis for thyroid cancer, Translational Research. 166 (2015) 188-195. https://doi.org/10.1016/j.trs1.2015.01.005.

[11] C. m. Willis, L. e. Britton, M. a. Swindells, E. m. Jones, A. e. Kemp, N. 1. Muirhead, A. Gul, R. n. Matin, L. Knutsson, M. Ali, Invasive melanoma in vivo can be distinguished from basal cell carcinoma, benign naevi and healthy skin by canine olfaction: a proof-ofprinciple study of differential volatile organic compound emission, Br J Dermatol. 175 (2016) 1020-1029. https://doi.org/10.1111/bjd.14887.

[12] J. Huang, S. Kumar, G.B. Hanna, Investigation of C3-C10 aldehydes in the exhaled breath of healthy subjects using selected ion flow tube-mass spectrometry (SIFT-MS), Journal of Breath Research. 8 (2014) 037104. https://doi.org/10.1088/1752$7155 / 8 / 3 / 037104$.

[13] P. Španěl, D. Smith, Progress in SIFT-MS: Breath analysis and other applications, Mass Spectrom. Rev. 30 (2011) 236-267. https://doi.org/10.1002/mas.20303.

[14] A. Catala, C. Levasseur-Garcia, M. Pagès, J.-L. Schaff, U. Till, L. Vitola Pasetto, M. Hausberger, H. Cousillas, F. Violleau, M. Grandgeorge, Prediction and detection of human epileptic seizures based on SIFT-MS chemometric data, Sci Rep. 10 (2020) 18365. https://doi.org/10.1038/s41598-020-75478-8.

[15] S. Grassin-Delyle, C. Roquencourt, P. Moine, G. Saffroy, S. Carn, N. Heming, J. Fleuriet, H. Salvator, E. Naline, L.-J. Couderc, P. Devillier, E.A. Thévenot, D. Annane, 
Metabolomics of exhaled breath in critically ill COVID-19 patients: A pilot study, EBioMedicine. 63 (2021) 103154. https://doi.org/10.1016/j.ebiom.2020.103154.

[16] V.S. Langford, C.J. Reed, D.B. Milligan, M.J. McEwan, S.A. Barringer, W.J. Harper, Headspace Analysis of Italian and New Zealand Parmesan Cheeses, Journal of Food Science. 77 (2012) C719-C726. https://doi.org/10.1111/j.1750-3841.2012.02730.x.

[17] V. Langford, J. Gray, B. Foulkes, P. Bray, M.J. McEwan, Application of Selected Ion Flow Tube-Mass Spectrometry to the Characterization of Monofloral New Zealand Honeys, Journal of Agricultural and Food Chemistry. 60 (2012) 6806-6815. https://doi.org/10.1021/jf3025002.

[18] J. Brändle, K.J. Domig, W. Kneifel, Relevance and analysis of butyric acid producing clostridia in milk and cheese, Food Control. 67 (2016) 96-113. https://doi.org/10.1016/j.foodcont.2016.02.038.

[19] M.E. Dolch, C. Hornuss, C. Klocke, S. Praun, J. Villinger, W. Denzer, G. Schelling, S. Schubert, Volatile organic compound analysis by ion molecule reaction mass spectrometry for Gram-positive bacteria differentiation, European Journal of Clinical Microbiology \& Infectious Diseases. 31 (2012) 3007-3013. https://doi.org/10.1007/s10096-012-1654-2.

[20] K. Sovová, V. Shestivska, P. Španěl, Real-Time Quantification of Traces of Biogenic Volatile Selenium Compounds in Humid Air by Selected Ion Flow Tube Mass Spectrometry, Analytical Chemistry. 84 (2012) 4979-4983. https://doi.org/10.1021/ac300609m.

[21] A. Gibson, L. Malek, R.F.H. Dekker, B. Ross, Detecting volatile compounds from Kraft lignin degradation in the headspace of microbial cultures by selected ion flow tube mass spectrometry (SIFT-MS), Journal of Microbiological Methods. 112 (2015) 40-45. https://doi.org/10.1016/j.mimet.2015.03.008.

[22] M.C. Lemfack, S.R. Ravella, N. Lorenz, M. Kai, K. Jung, S. Schulz, B. Piechulla, Novel volatiles of skin-borne bacteria inhibit the growth of Gram-positive bacteria and affect quorum-sensing controlled phenotypes of Gram-negative bacteria, Systematic and Applied Microbiology. (2016). https://doi.org/10.1016/j.syapm.2016.08.008.

[23] F. Biasioli, C. Yeretzian, T.D. Märk, J. Dewulf, H. Van Langenhove, Direct-injection mass spectrometry adds the time dimension to (B)VOC analysis, TrAC Trends in Analytical Chemistry. 30 (2011) 1003-1017. https://doi.org/10.1016/j.trac.2011.04.005.

[24] D. Smith, M.J. McEwan, P. Španěl, Understanding Gas Phase Ion Chemistry Is the Key to Reliable Selected Ion Flow Tube-Mass Spectrometry Analyses, Anal. Chem. 92 (2020) 12750-12762. https://doi.org/10.1021/acs.analchem.0c03050.

[25] M. Ghislain, N. Costarramone, J. Sotiropoulos, T. Pigot, R. Van Den Berg, S. Lacombe, M. Le Bechec, Direct analysis of aldehydes and carboxylic acids in the gas phase by negative ionization selected ion flow tube mass spectrometry: Quantification and modelling of ion-molecule reactions, Rapid Commun Mass Spectrom. 33 (2019) 16231634. https://doi.org/10.1002/rcm.8504.

[26] P. Španěl, M. Pavlik, D. Smith, Reactions of $\mathrm{H} 3 \mathrm{O}+$ and $\mathrm{OH}-$ ions with some organic molecules; applications to trace gas analysis in air, International Journal of Mass Spectrometry and Ion Processes. 145 (1995) 177-186. https://doi.org/10.1016/01681176(95)04164-G.

[27] L. Vitola Pasetto, V. Simon, R. Richard, J.-S. Pic, F. Violleau, M.-H. Manero, Aldehydes gas ozonation monitoring: Interest of SIFT/MS versus GC/FID, Chemosphere. 235 (2019) 1107-1115. https://doi.org/10.1016/j.chemosphere.2019.06.186. 
[28] P. Spanel, D. Smith, Reactions of Hydrated Hydronium Ions and Hydrated Hydroxide Ions with Some Hydrocarbons and Oxygen-Bearing Organic Molecules, J. Phys. Chem. 99 (1995) 15551-15556. https://doi.org/10.1021/j100042a033.

[29] D. Hera, V. Langford, M. McEwan, T. McKellar, D. Milligan, Negative Reagent Ions for Real Time Detection Using SIFT-MS, Environments. 4 (2017) 16. https://doi.org/10.3390/environments4010016.

[30] S. Williams, M.F. Campos, A.J. Midey, S.T. Arnold, R.A. Morris, A.A. Viggiano, Negative Ion Chemistry of Ozone in the Gas Phase, J. Phys. Chem. A. 106 (2002) 9971003. https://doi.org/10.1021/jp012929r.

[31] L. Vitola Pasetto, R. Richard, J.-S. Pic, M.-H. Manero, F. Violleau, V. Simon, Ozone Quantification by Selected Ion Flow Tube Mass Spectrometry: Influence of Humidity and Manufacturing Gas of Ozone Generator, Anal. Chem. 91 (2019) 15518-15524. https://doi.org/10.1021/acs.analchem.9b03337.

[32] M. Ghislain, N. Costarramone, T. Pigot, M. Reyrolle, S. Lacombe, M. Le Bechec, High frequency air monitoring by selected ion flow tube-mass spectrometry (SIFT-MS): Influence of the matrix for simultaneous analysis of VOCs, $\mathrm{CO} 2$, ozone and water, Microchemical Journal. (2019) 104435. https://doi.org/10.1016/j.microc.2019.104435.

[33] P. Španěl, D. Smith, Quantitative selected ion flow tube mass spectrometry: The influence of ionic diffusion and mass discrimination, Journal of the American Society for Mass Spectrometry. 12 (2001) 863-872. https://doi.org/10.1016/S10440305(01)00253-7.

[34] P. Spanel, D. Smith, Influence of water vapour on selected ion flow tube mass spectrometric analyses of trace gases in humid air and breath, Rapid Communications in Mass Spectrometry. 14 (2000) 1898-1906. https://doi.org/10.1002/10970231(20001030)14:20<1898::AID-RCM110>3.0.CO;2-G.

[35] M.J. Frisch, G.W. Trucks, H.B. Schlegel, G.E. Scuseria, M.A. Robb, J.R. Cheeseman, G. Scalmani, V. Barone, B. Mennucci, G.A. Petersson, H. Nakatsuji, M. Caricato, X. Li, H.P. Hratchian, A.F. Izmaylov, J. Bloino, G. Zheng, J.L. Sonnenberg, M. Hada, M. Ehara, K. Toyota, R. Fukuda, J. Hasegawa, M. Ishida, T. Nakajima, Y. Honda, O. Kitao, H. Nakai, T. Vreven, Jr. Montgomery J.A., J.E. Peralta, F. Ogliaro, M. Bearpark, J.J. Heyd, E. Brothers, K.N. Kudin, V.N. Staroverov, R. Kobayashi, J. Normard, K. Raghavachari, A. Rendell, J.C. Burant, S.S. Iyengar, J. Tomasi, M. Cossi, N. Rega, J.M. Millam, M. Klene, J.E. Knox, J.B. Cross, V. Bakken, C. Adamo, J. Jaramillo, R. Gomperts, R.E. Stratmann, O. Yazyev, A.J. Austin, R. Cammi, C. Pomelli, J.W. Ochterski, R.L. Martin, K. Morokuma, V.G. Zakrzewski, G.A. Voth, P. Salvador, J.J. Dannenberg, S. Dapprich, A.D. Daniels, Ö. Farkas, J.B. Foresman, J.V. Ortiz, J. Cioslowski, D.J. Fox, Gaussian 09, Revision D.01, (2009).

[36] T. Su, W.J. Chesnavich, Parametrization of the ion-polar molecule collision rate constant by trajectory calculations, The Journal of Chemical Physics. 76 (1982) 51835185. https://doi.org/10.1063/1.442828.

[37] P. Španěl, D. Smith, SIFT studies of the reactions of $\mathrm{H} 3 \mathrm{O}+, \mathrm{NO}+$ and $\mathrm{O} 2+$ with a series of volatile carboxylic acids and esters, International Journal of Mass Spectrometry and Ion Processes. 172 (1998) 137-147. https://doi.org/10.1016/S0168-1176(97)00246-2.

[38] G.J. Francis, D.B. Milligan, M.J. McEwan, Gas-Phase Reactions and Rearrangements of Alkyl Esters with $\mathrm{H} 3 \mathrm{O}+$, NO+, and O2 $\bullet+$ : A Selected Ion Flow Tube Study, J. Phys. Chem. A. 111 (2007) 9670-9679. https://doi.org/10.1021/jp0731304. 